\title{
Neue ${ }^{14} \mathrm{C}$-Daten zur jüngeren Dryaszeit in den ecuadorianischen Anden
}

\author{
Klaus Heine \& Mebus A. GeyH*)
}

Heine, K. \& Geyh, M. A. (2002): Neue ${ }^{14}$ C-Daten zur jüngeren Dryaszeit in den ecuadorianischen Anden. Eiszeitalter und Gegenwart, 51: 33-50; Hannover 2002.

Keywords: Younger Dryas, glacier advance, Ecuadorian Andes, ${ }^{14} \mathrm{C}$ age related problems.

Kurzfassung: Glaziale Sedimente und Formen der tropischen Anden enthalten Paläoklima-Archive. Für die Jüngere Dryaszeit (Younger Dryas $=$ YD) besteht eine kontroverse Diskussion bezüglich der Gletschervorstöße. Im Gebiet des Papallacta-Passes (Ostkordilleren in Ecuador) wird ein Gletschervorstoß durch Till und Moränen dokumentiert. Die glazialen Sedimente werden unter- und überlagert von Tephrahorizonten, Torf und anderen Sedimenten, die reich an organischem Material sind. Radiokarbon-Altersbestimmungen werden somit möglich. $29{ }^{14} \mathrm{C}$-Alter zu diesem Gletschervorstoß publizierten CLAPPERTON et al. (1997); weitere $29{ }^{14} \mathrm{C}$-Alter werden hier vorgestellt. Die Daten belegen sowohl feuchte (= Gletschervorstoß) als auch trockene (= Gletscherabschmelzen) Klimabedingungen während der Jüngeren Dryaszeit. Unter Berücksichtigung der kalibrierten ${ }^{14} \mathrm{C}$-Alter ergibt sich ein Gletschervorstoß in der zweiten Hälfte der Jüngeren Dryaszeit und zu Beginn des Holozäns. An dem Beispiel wird belegt, daß die Interpretation $\operatorname{der}^{14} \mathrm{C}$-datierten Jüngere Dryas-zeitlichen Gletschervorstöße mit größter Sorgfalt vorgenommen werden muß.

\section{$\left[\mathrm{New}{ }^{14} \mathrm{C}\right.$ dates of Younger Dryas age in the Ecuadorian Andes]}

\begin{abstract}
The tropical Andes contain archives of late Quaternary climate change in glacial deposits. There is much debate about the Younger Dryas (YD) interval with respect to glacier advances. A glacier advance is documented by tills and moraines in the Papallacta Pass area of the eastern Andean Cordillera in Ecuador. The glacial deposits are underlain and overlain by tephras, peat and other deposits rich in organic matter, thus allowing radiocarbon age determinations. $29{ }^{14} \mathrm{C}$ ages published by CLAPPERTON et al. (1997) and $29{ }^{14} \mathrm{C}$ ages presented in this study have been interpreted to show both wet (= glacier advance) and dry (= glacier retreat) conditions during the YD. A glacier advance occur-
\end{abstract}

\footnotetext{
*)Anschrift der Verfasser: Prof. Dr. K. Heine, Geographisches Institut, Universität, D-93040 Regensburg; Prof. Dr. M.A. Geyh, Institut für Geowissenschaftliche Gemeinschaftsaufgaben, Postf. 510153, D-30631 Hannover.
}

red during the latter half of the YD and the early Holocene. The case shows that the interpretation of ${ }^{14} \mathrm{C}$ dated YD glacier advances has to be elaborated very carefully.

\section{Einleitung}

Es ist heute bekannt, daß die letzteiszeitlichen Maximalstände der Gebirgs- und der kontinentalen Vergletscherungen asynchron verliefen (GILLespie \& Molnar 1995; Heine 1983; Clark et al. 1993). In den Gebirgen von N- und S-Amerika, Hawaii, Asien und Neuseeland ereigneten sich die maximalen Gletschervorstöße vor dem eigentlichen LGM (= last glacial maximum, um ca. 18.000 ${ }^{14} \mathrm{C}$-Jahre vor heute), nämlich während verschiedener Zeitabschnitte zwischen 115.000 und 30.000 a BP, als niedrige Temperaturen und hohe Niederschläge besonders günstig für das Gletscherwachstum waren (z.B. MIS $4=$ marine isotope stage 4 , ca. 73.000-58.000 a BP). Unterschiede in der Zeit der Gletschervorstöße in den verschiedenen Gebirgen weisen darauf hin, daß alpine Gletscher nicht nur vom globalen Klimageschehen abhängig sind, sondern auch in gleicher Weise vom regionalen Klima. In einem engeren zeitlichen Maßstab trifft dies auch für das Spätglazial und Frühholozän, also die Zeit zwischen rund 14.000 und $9000{ }^{14} \mathrm{C}$-Jahren, zu. Außerdem dokumentiert das Verhalten größerer Gletscher im Gegensatz zu kleineren nur die langfristigen, einschneidenden Klimaveränderungen. Es verwundert daher sehr, daß auch heute noch immer wieder sogenannte Jüngere Dryas-zeitliche Gletschervorstöße postuliert und korreliert werden, obgleich dafür keine hinreichenden Belege vorhanden sind. Allein aus der Vorstellung heraus, $\mathrm{daß}$ der Klimasturz im nordatlantischen Bereich sich auch weltweit bemerkbar gemacht haben muß und daß damit weltweit ein Vorrücken der Gebirgsgletscher einherging, werden spätglaziale Gletschervorstöße vorschnell der YD zugeschrieben. Zahlreiche Belege aus allen Erdteilen lassen sich dafür anführen (z.B. Denton \& Hendy 1994; DENTON et al. 1999). 
Autoren, die eine kritische und vorsichtige Interpretation der - oft spärlichen - Paläoklimadaten fordern (z.B. Heine 1983; J. T. Heine 1993), werden wenig beachtet. Über viele Jahre wurden Ergebnisse der Eisbohrkerne von Grönland und aus der Antarktis hinsichtlich der spätglazialen Klimaschwankungen korreliert, ohne daß dafür genügend gesicherte absolute Daten vorlagen. Erst kürzlich wurde aufgrund eines neuerlichen Vergleichs der Eisbohrkerne von Vostok und Byrd/ Antarktis und GRIP/Grönland darauf aufmerksam gemacht (RAYNAUD et al. 2000), daß eine kalte spätglaziale Klimaschwankung der Antarktis, die bisher der YD zugeschrieben wurde, nicht der YD des nordatlantischen Raumes entspricht. RÜHI.EMANN et al. (1999) weisen darauf hin, daß sich auch die SST (sea surface temperature) des tropischen und des Nord-Atlantik während des Spätglazials entgegengesetzt verhielten.

Es ist eine große Herausforderung für die Paläoklimatologen, Zeit und Mechanismus der abrupten Klimaänderungen auf der Nord- und der Südhemisphäre während des Spätglazials zu identifizieren. Dies ist nur möglich, indem detaillierte paläoklimatische Archive aus den Tropen und der S-Hemisphäre ausgewertet werden. Viele Fragen sind bisher unbeantwortet (vgl. Denton 2000):

- Sind die abrupten Änderungen, die in den Grönland-Eiskernen zu beobachten sind, regional oder global?

Erwärmte sich die Süd-Hemisphäre früher?

Oder erwärmten sich beide Hemisphären plötzlich und gleichzeitig?

Oder erwärmte sich die eine Hemisphäre plötzlich und die andere allmählich?

Lassen sich die YD-Kälteschwankung und die Bölling-Warmperiode auf der Süd-Hemisphäre belegen? (vgl. JouzEL et al. 1995).

- Oder zeigen beide Hemisphären während der Bölling/Alleröd-YD-Zeit Klimaschwankungen mit gegenläufigem Trend?

Um die Klärung dieser Fragen voranzutreiben, werden detaillierte zeitliche Angaben zum spätglazialen/frühholozänen Gletscherverhalten der ecuadorianischen Anden mitgeteilt. In Verbindung mit neueren Forschungen über die Klima-Gletscher-Beziehungen in den Tropen und die hohe Empfindlichkeit, mit der tropische Gletscher auf Temperaturänderungen reagieren (KASER et al. 1996a; 1996b), tragen sie zu einer differenzierteren Interpretation der Jüngere Dryas-zeitlichen Gletscherschwankungen in den Tropen bei.
Große Probleme bereiten immer noch viele ${ }^{14} \mathrm{C}$ Alter, da methodische Annahmen für deren Bestimmung zugrunde gelegt werden, die nicht immer erfüllt sind. Bisher unberücksichtigt blieb meist, daß der Reservoireffekt der Meere sich möglicherweise im Jungquartär geändert hat (SIKES et al. 2000); dies führt zu Fehlern bei der absoluten Datierung der Meeressedimente und erschwert Korrelationen zwischen marinen und terrestrischen Sedimenten und Eisbohrkernen.

\subsection{Ursachen der YD}

Die YD wird in die Zeit zwischen 11.000 und $10.000{ }^{14} \mathrm{C}$-Jahre BP datiert. In den letzten Jahren wurde durch Kalibrierung der ${ }^{14} \mathrm{C}$-Daten Anfang und Ende der YD absolut angegeben. Folgende Alter werden genannt: TAYLOR et al. (1993): 12.800-11.600 cal BP; RÜHLEMANN et al. (1999): 12.900-11.600 cal BP; GosLAR et al. (2000): 12.70011.500 cal BP. Das Ende der YD (Termination YD/PB) bestimmen ANDrEs \& LITT (2000) auf 11.560 bis 11.590 cal BP für Mitteleuropa aufgrund der Ergebnisse des DFG-Projekts „Wandel der Geo-Biosphäre während der letzten 15000 Jahre, kontinentale Sedimente als Ausdruck sich verändernder Umweltbedingungen".

Nach Hostetler et al. (2000) existierten um $11.000{ }^{14} \mathrm{C}$-Jahre BP große Seen (Lake Agassiz, vgl. Colman et al. 1994) in Zentral- und OstNordamerika am Südrand des Laurentischen Eisschildes. Diese haben das Klima beeinflußt. Durch mesoskalige atmosphärische Rückkopplungen war die Zufuhr der Feuchtigkeit auf den laurentischen Eisschild verringert und zwar in Zeiten, in denen der ausgedehnte kalte Lake Agassiz existierte, im Vergleich zu den Zeiten mit niedrigen Seeständen, in denen nur kleine Seen vorhanden waren. Die daraus resultierenden Änderungen der Massenbilanz des Eisschildes hat zu Fluktuationen seiner Ränder geführt, die wiederum die Abflußwege des Wassers zum Nord-Atlantik beeinflußten. Ein zurückweichender Eisrand während Zeiten mit hohem Seespiegel konnte den Abflußweg vom Lake Agassiz zum Nord-Atlantik öffnen. Ein nachfolgendes Vorrücken des Eisrandes als Folge größerer Feuchtigkeitszufuhr bei niedrigem Seespiegel konnte den Abflußweg wieder versperren und dadurch den Abfluß in den Nord-Atlantik reduzieren (DE VERNAL et al. 1996; vgl. auch Colman et al. 1994).

Man vermutet, daß die YD mit einer großen Verschiebung der nordatlantischen thermohalinen 
Zirkulation, die fast zu LGM-Verhältnissen führte, verbunden ist. Diese Verschiebung kann durch die Schmelzwasser und Eissurges ausgelöst worden sein (vgl. Anderson 1997). Goslar et al. (2000) bemerken, daß während der YD die Tiefenzirkulation der Ozeane nicht wesentlich von den gegenwärtigen Verhältnissen abwichen. Daher kann die Ursache für die YD sehr wohl in dem Schmelzwasser-Abfluß liegen.

\subsection{Problemstellung}

Im Zuge des Ausbaus der Papallacta-Paßstraße zwischen Quito und dem Amazonas-Tiefland waren in den Jahren 1990 und 1991 ideale Aufschlußverhältnisse in der Ostkordillere der Anden im Paßbereich zwischen 3600 und $4080 \mathrm{~m}$ Höhe gegeben (Abb. 1 u. 2). Die Geländeaufnahmen zeigten, daß ein spätglazialer bis frühholozäner Gletscher (Gruppe VI) im Paßgebiet oberhalb $4000 \mathrm{~m} \mathrm{NN}$ existierte. Aufgrund umfangreicher Gelände- und Laborbefunde können HEINe (1995a) und HeIne \& HeIne (1996) die Moränen einem Gletschervorstoß an der Wende YD/ Holozän zuordnen. Vierzehn ${ }^{14} \mathrm{C}$-Daten aus dem Papallacta-Gebiet belegen ein maximales Alter des Gletschervorstoßes um bzw. nach $10.500{ }^{14} \mathrm{C}$-Jah-

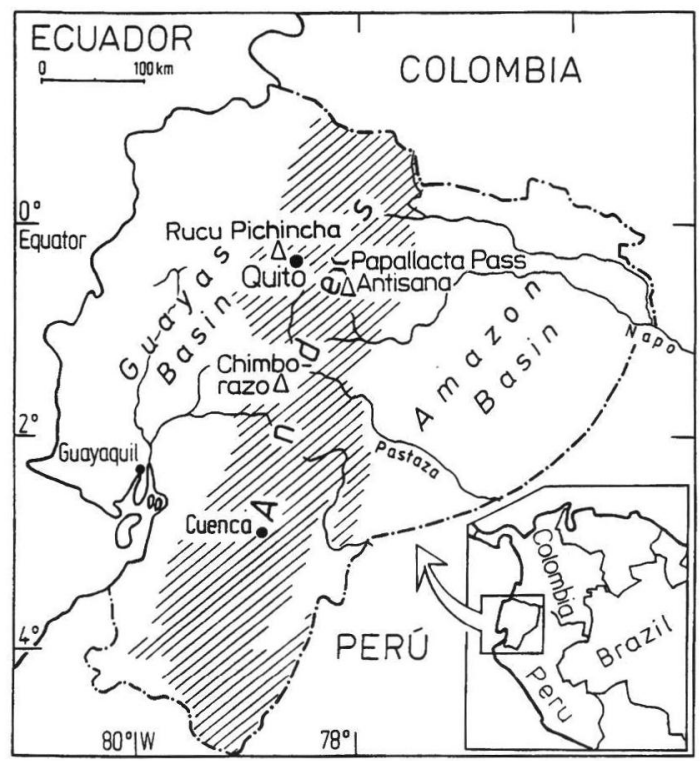

Abb. 1: Karte von Ecuador.

Fig. 1: Location map.

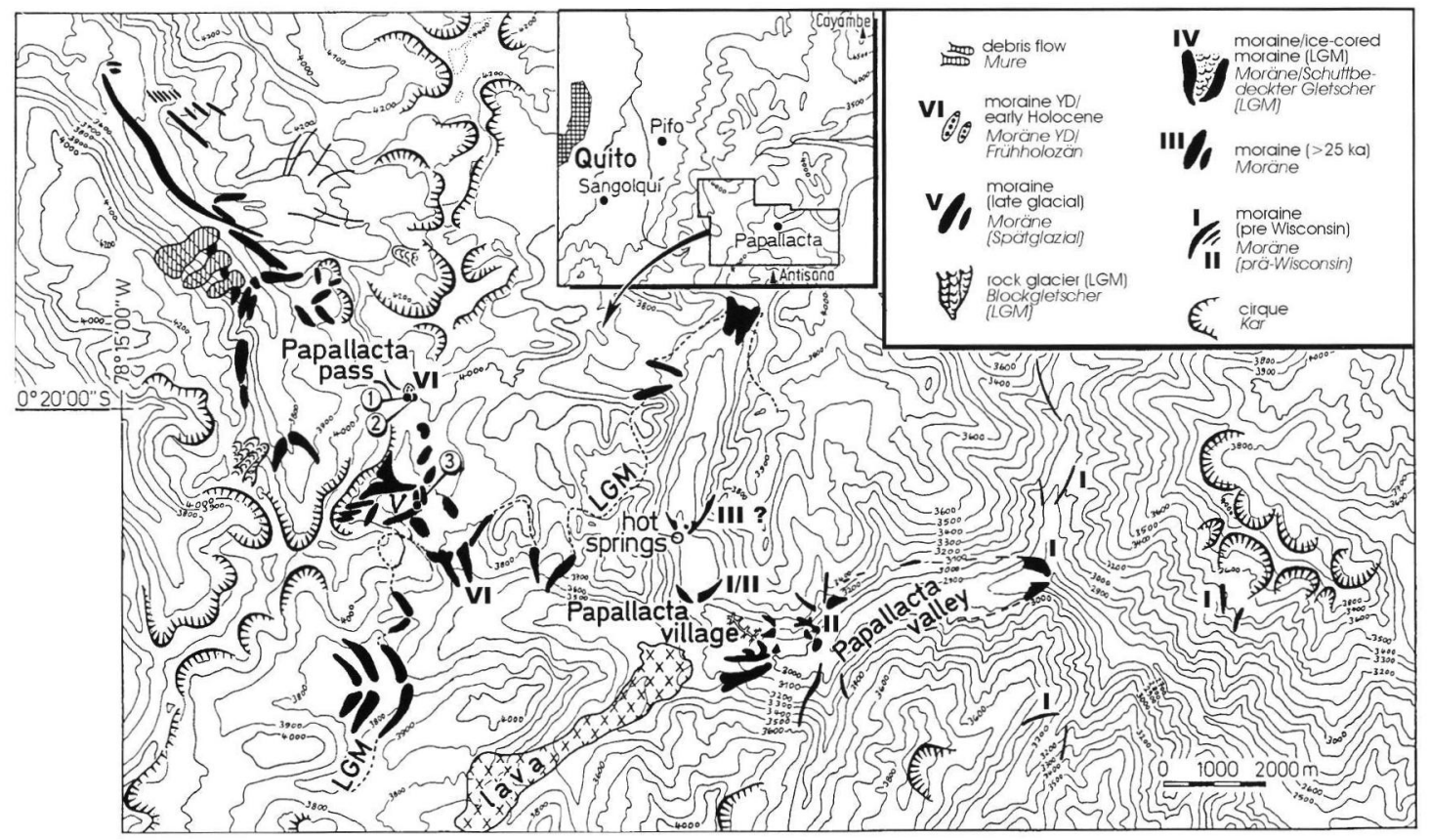

Abb. 2: Glazialmorphologische Skizze des Papallacta-Tals (nach HeNE 1995a). (1) und (2): Lage der Profile der Moräne M VI; (3): Lage der Profile der Moräne V. 
ren BP und - in Verbindung mit Daten vom Pichincha-Vulkan - ein minimales Alter von $>9000$ ${ }^{14} \mathrm{C}$-Jahren BP. Heine (1995a) und Heine \& HeINE (1996) datieren den Gletschervorstoß nicht unmittelbar in die Phase der Jüngeren Dryaszeit, sondern gegen Ende der YD und zu Beginn des Holozäns. Nachdem Clapperton von der Existenz dieser Moränen erfahren hat, wurden sie von ihm und einer Arbeitsgruppe erneut bearbeitet und datiert. Diese Moränen der Gruppe VI bezeichnen Clapperton et al. (1997) als 'Potrerillos Glacier Advance', den sie zwischen 10.855 und 10.035 ${ }^{14} \mathrm{C}$-Jahre BP datieren und als Vorstoß deklarieren, der der europäischen Jüngeren Dryaszeit zuzuordnen ist. Clapperton et al. (1997) bestimmen das Maximalalter der Moränengruppe M VI, indem sie gewichtete mittlere Alter aus mehreren ${ }^{14} \mathrm{C}$-Daten verschiedener Profile und Horizonte berechnen. Das angewandte Verfahren ist mathematisch nicht haltbar und wird daher von GEYH (2000) nachdrücklich kritisiert.

Um das Alter der Moränengruppe M VI genauer zu erfassen, wurden zusätzliche ${ }^{14} \mathrm{C}$-Altersbestimmungen ausgeführt und eine weitere Auswertung der Gelände- und Laborbefunde vorgenommen. Die Ergebnisse werden vor dem Hintergrund der heutigen Kenntnisse über die YD nachfolgend diskutiert.

\section{Befunde}

\subsection{Das Arbeitsgebiet}

Die Kordilleren der Anden erheben sich in Ecuador mehr als $4000 \mathrm{~m}$ über die Tiefländer von Guayas im Westen und Amazoniens im Osten. Die Gebirgsketten verlaufen in nordsüdlicher Richtung und werden von Stratovulkanen, die oft Höhen von mehr als $5000 \mathrm{~m}$ erreichen, gekrönt (Abb. 1). Intramontane Becken und Plateaus trennen die Hauptgebirgszüge. Clapperton (1987a) gibt eine ausführliche Beschreibung von Relief, Klima, Gletschern und Glazialmorphologie des Raumes. Physisch-geographische Daten zum Gebiet des Papallacta-Passes geben Clapperton et al. (1997).

Die Vergletscherungen der ecuadorianischen Anden werden seit über 100 Jahren diskutiert (u.a. Wolf 1892; SAUER 1971; Hastenrath 1981; LAUer \& Rafiqpoor 1986; Clapperton 1993; Heine 1995a). Clapperton (1985; 1987a; 1987b; 1990; 1993; Clapperton \& McEwAn 1985) erarbeitet eine Glazialchronologie für Ecuador, in der die oft fundamentalen Irrtümer früherer Studien gezeigt und korrigiert werden. HeINE (1995a; 1995b) kann darlegen, daß auch die von CLAPPERTON (1993) vorgestellte Chronostratigraphie in wesentlichen Punkten (z.B. der Ausdehnung der LGMzeitlichen Gletscher) einer Revision bedarf.

\subsection{Spätglaziale Gletschergeschichte am Papallacta-Paß}

Das Papallacta-Tal und der Papallacta-Paß (Abb. 2) befinden sich nördlich und nordwestlich des Antisana-Vulkans. Die glazialen Formen und Sedimente weisen auf wiederholte Vergletscherungen. Moränen (M I) belegen, daß ein pleistozäner Gletscher im Papallacta-Tal bis $2800 \mathrm{~m}$ Höhe hinab reichte (HeINE 1995a). Diese am tiefsten liegende Moräne M I wird in das MIS 6 (marine isotope stage 6) oder älter datiert (HEINE 1995a). Zwischen der Moräne M I und dem Papallacta$\mathrm{Paß}$ (Abb. 2) ist eine Sequenz aus zahlreichen, unterschiedlich alten Moränen ausgebildet, die Gletscherschwankungen seit dem MIS 6 dokumentieren. Die Moränen der Gruppe M IV, die dem LGM (last glacial maximum, ca. $18.000{ }^{14} \mathrm{C}$ Jahre vor heute) angehören, liegen in 3700-3900 m Höhe.

Die Moränen der hier zur Diskussion stehenden Gruppe M VI werden von der Paßstraße in Höhen oberhalb von $4000 \mathrm{~m} \mathrm{NN}$ angeschnitten (Foto 1 und 2). Der Till füllt eine flache Mulde aus; er ist unverwittert, weder sortiert noch eingeregelt, ungegliedert und bis zu $6 \mathrm{~m}$ mächtig. Gerundete Blöcke können einen Durchmesser bis über 0,5 $\mathrm{m}$ haben. Das gesamte Profil zeigt folgenden Aufbau: Über den größtenteils durch Gletschereis abgeschliffenen Vulkangesteinen liegt ein 20 bis $30 \mathrm{~cm}$ mächtiger Horizont aus Schottern und Periglazialschutt, der zu den seitlichen Hängen hin auskeilt. Darüber folgt eine Wechsellagerung aus Tephren und Mudden. Mindestens 7 dünne Bänder sind reich an organischem Material. Die Sedimentation dieses etwa $70 \mathrm{~cm}$ dicken Schichtpakets erfolgte durch starke Abspülung und Umlagerung. Tephralagen in situ werden nicht beobachtet, obgleich einige weißgraue Tuffbändchen fast durchgehend zu verfolgen sind. Die Mudden weisen auf Phasen morphologischer Stabilität hin.

Die Datierung des organischen Materials einiger Horizonte (Profil A) ergab folgende ${ }^{14} \mathrm{C}$-Alter (a BP) (Abb. 3): Das älteste Muddebändchen hat ein ${ }^{14} \mathrm{C}$-Alter von $12.140 \pm 80$ a $\mathrm{BP}(\mathrm{Hv} 17062)$ und 


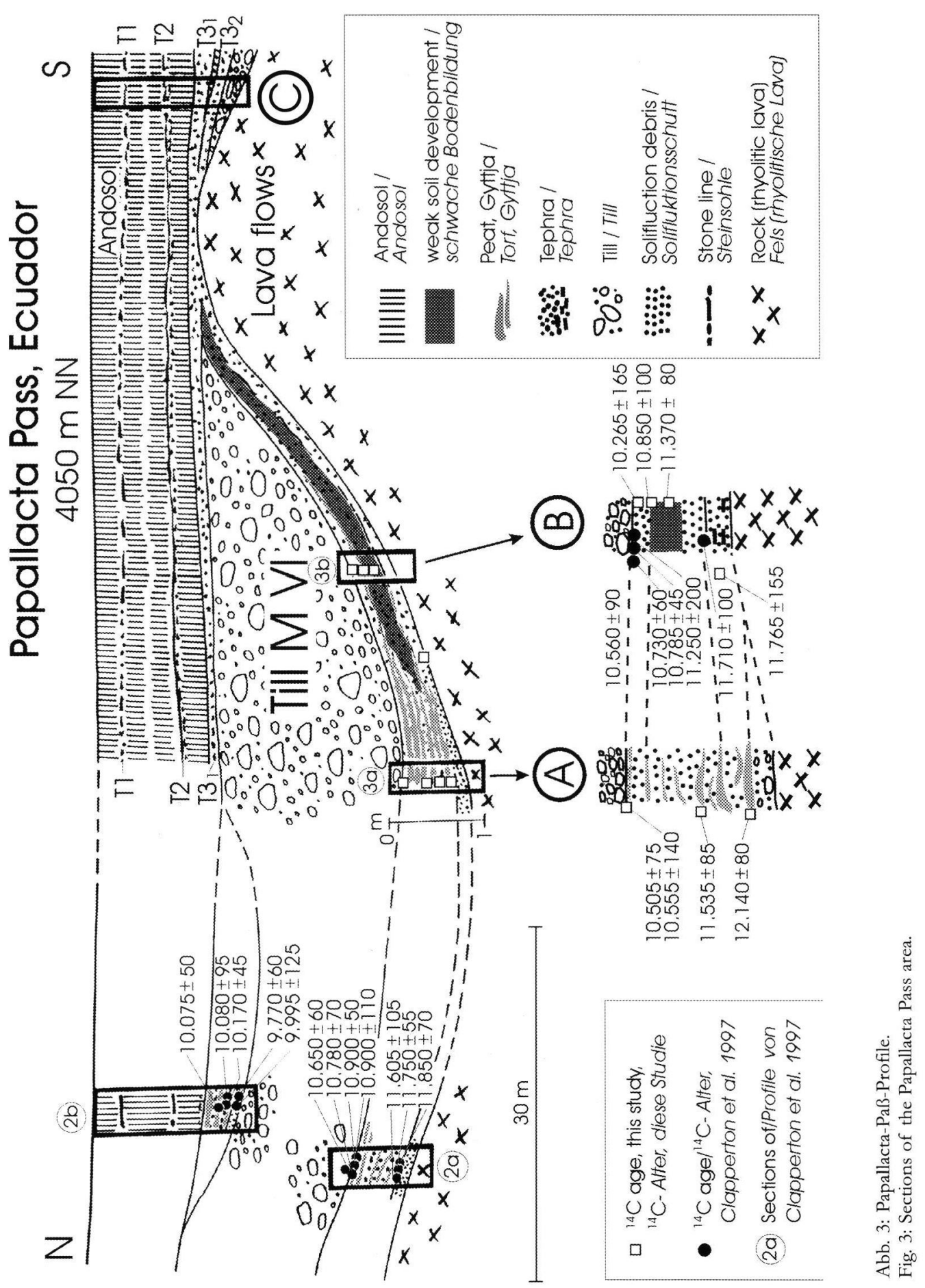

zeigt damit den Beginn der spätglazialen Wiederbesiedlung dieser Gebiete durch die Vegetation an. Der Horizont aus Schottern und Periglazialschutt darunter ist entsprechend älter. Ein zwei- tes ${ }^{14} \mathrm{C}$-Alter von $11.535 \pm 85$ a $\mathrm{BP}(\mathrm{Hv} 23019)$ in Verbindung mit den ${ }^{14} \mathrm{C}$-Altern der hangenden Muddeschicht von 10.555 \pm 140 a BP (Hv 23020) und 10.505 \pm 75 a BP (Hv 17063) belegt die konti- 


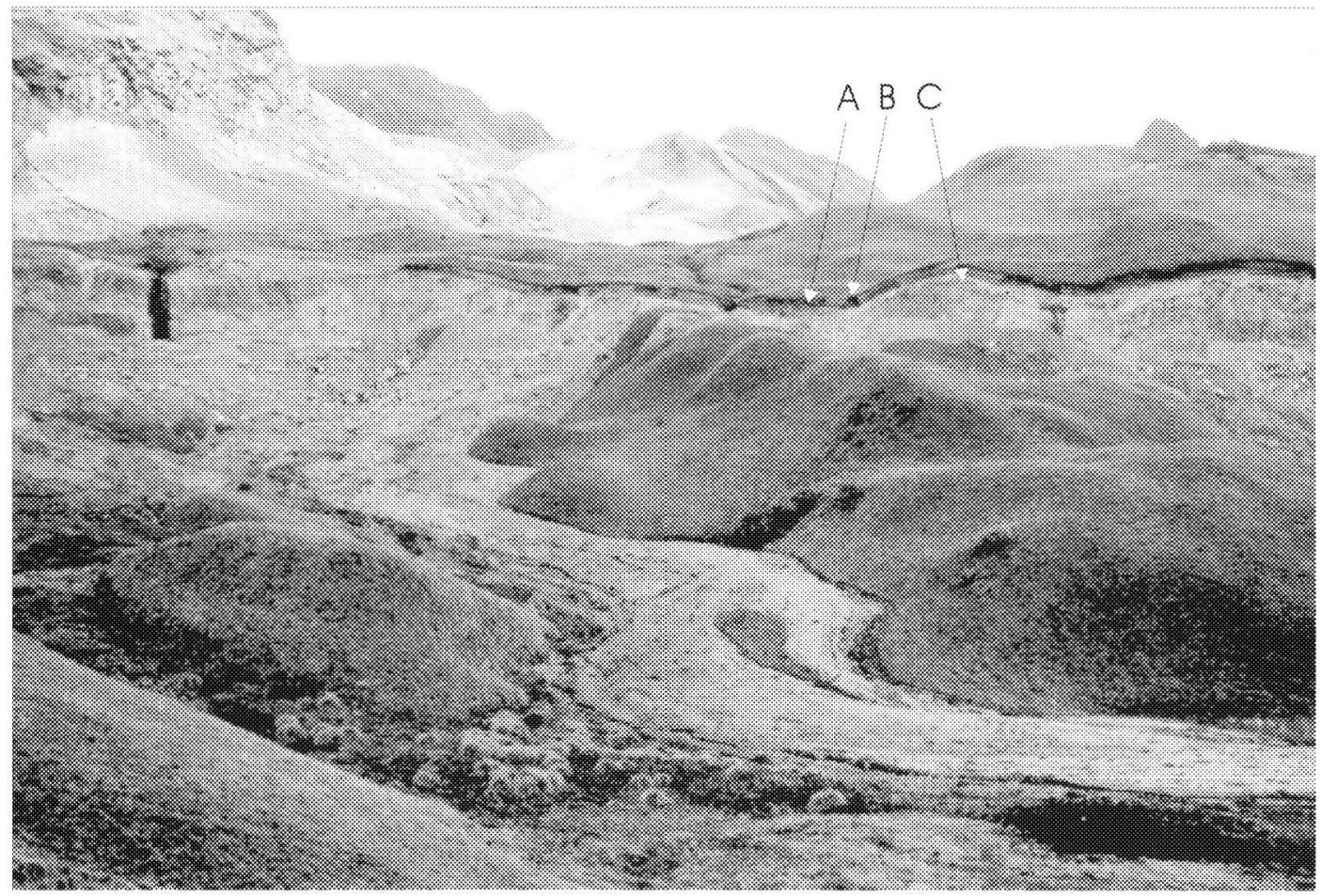

Foto 1: Papallacta-Paß-Gebiet in rund 4000-4100 m NN. Die Straßeneinschnitte zeigen den Aufbau der Moränen sowie der liegenden und hangenden Schichten auf mehreren $100 \mathrm{~m}$ Länge. Die Pfeile markieren die Profile A,B und C der Abbildung 3 (siehe auch Foto 2). Foto: HeINe, 20.01.1990.

Foto 1: Papallacta Pass area, ca. $4000-4100 \mathrm{~m}$ a.s.l. The road-cuts which are several $100 \mathrm{~m}$ long, show the tills and the tephra, peat and soil layers on top and beneath the glacial deposits. The arrows mark the sections $\mathrm{A}, \mathrm{B}$ and $\mathrm{C}$ of Figure 3 (see also Photo 2). Photo: Heine, 20.01.1990).

nuierliche Sedimentation der Wechsellagerung aus Tephra und Mudden bis vor rund $10.500{ }^{14} \mathrm{C}$-Jahren BP. Die Grenze der liegenden Sedimente zum Till dokumentiert, daß der Gletscher nicht erosiv tätig war, sondern Till in dem glazial geprägten Rundhöckerrelief aus Vulkaniten in den Senken zwischen den Felskuppen zurückließ.

Etwa $8 \mathrm{~m}$ südlich des zuvor genannten Profils A wurden die liegenden Schichten des Tills ebenfalls datiert (Profil B, Abb. 3). Unmittelbar unter dem Till hat eine Mudde ein ${ }^{14} \mathrm{C}$-Alter von $10.265 \pm 165$ a BP (Hv 23040). Ein Horizont aus Blattresten ergibt $10.850 \pm 100$ a BP (Hv 18068) und der darunter liegende Humushorizont $11.370 \pm 80$ a BP (Hv 23041). Damit ergänzen sich die Daten der Profile A und B.

Zwischen beiden Profilen hat organisches Material, das im liegenden Sand gefunden wurde, ein ${ }^{14} \mathrm{C}$-Alter von $11.765 \pm 155$ a BP (Hv 23042). Die- ser Horizont fügt sich mit seinem Alter ebenfalls in das Gesamtbild ein.

Ca. $20 \mathrm{~m}$ südlich des Profils $\mathrm{B}$ befindet sich das Profil C (Abb. 3). Hier bedeckt ein Schutt, der älter als die Wechsellagerung aus Tephren und Mudden ist, das Anstehende. Der Till der Profile $\mathrm{A}$ und $\mathrm{B}$ ist nicht mehr ausgebildet. Damit wird belegt, daß der Till der Moränengruppe M VI nur eine sehr begrenzte Verbreitung hat, was auf ein kleines Gletscherfeld schließen läßt.

Die Deckschichten der Profile A, B und C sind identisch. Es handelt sich um eine Wechsellagerung aus Tephraschichten und Andosols. Die Deckschichten lassen sich entlang der Straßeneinschnitte hangabwärts verfolgen. In $3870 \mathrm{~m}$ Höhe bedecken sie die Moränenwälle der Gruppe V, die ein Alter von $>13.000{ }^{14} \mathrm{C}$-Jahren BP haben (HeInE 1995a, 1995b; HeIne \& HeIne 1996). Aufgrund tephrostratigraphischer Korrelationen in Verbin- 


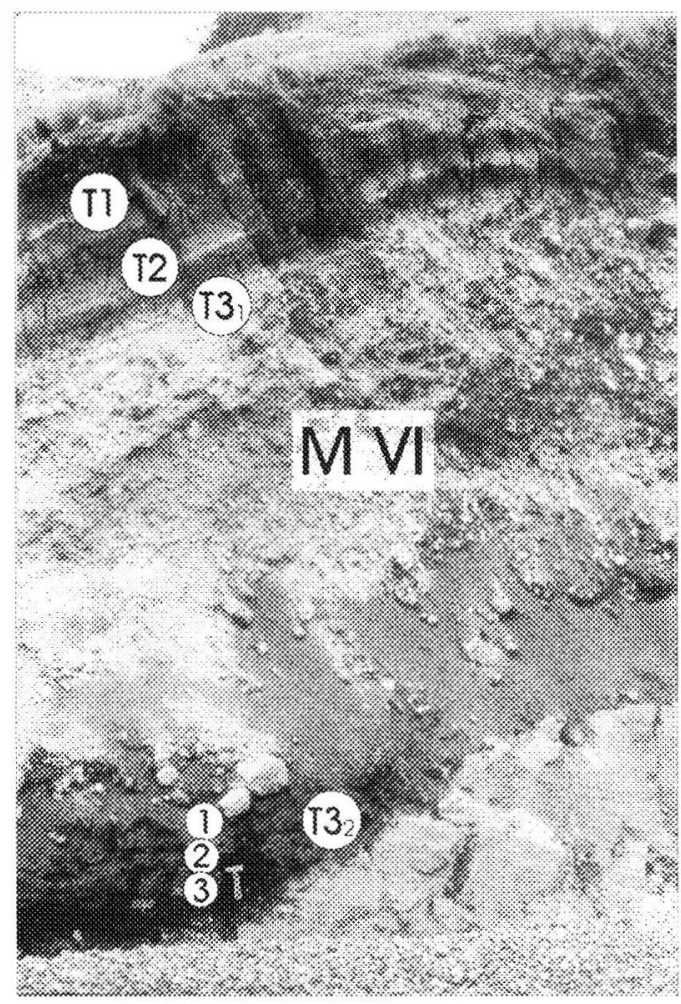

Foto 2: Papallacta-Paß-Gebiet in $4055 \mathrm{~m}$ NN. Aufschluß im Straßenanschnitt. Der unverwitterte Till der Moränengruppe M VI wird von Schotter, Sand, Torf und Tephra unterlagert (im Bild links unten). Die Deckschichten bestehen aus Tephra und Andoböden (im Bild oben links). Die Nummern zeigen die Entnahmestellen der Proben: (1) ECU $121(10.505 \pm 75-\mathrm{Hv} 17063 ; 10.555 \pm 140-\mathrm{Hv}$ 23020), (2) ECU 120 (11.535 \pm 85 - Hv 23019), (3) ECU 118 (12.140 $\pm 80-\mathrm{Hv}$ 17062). T1, T2, T3 und $\mathrm{T} 3=$ Tephralagen. Der Hammer neben dem Probenpunkt 3 gibt den Maßstab an. Foto: Heine, 21.01.1990.

Foto 2: Papallacta Pass area in $4055 \mathrm{~m}$ a.s.l. Section in roadcut. The unweathered till of moraine group M VI is underlain by gravel, sand, peat and tephra (below at left). The layers on top of the till are tephra and andosols (upper part, left). The numbers show the locations of samples: (1) ECU $121(10.505 \pm 75-\mathrm{Hv} 17063 ; 10.555 \pm 140-\mathrm{Hv}$ 23020), (2) ECU 120 (11.535 \pm 85 - Hv 23019), (3) ECU

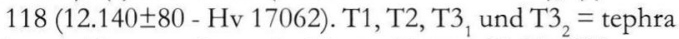
layers. Hammer for scale. Photo: Heine, 21.01.1990.

dung mit ${ }^{14} \mathrm{C}$-Altersbestimmungen (HEINE 1995a) werden die Deckschichten der Profile A, B und C dem Holozän zugeordnet. Die tephrostratigraphischen Korrelationen (Abb. 3) ergeben für die Tephrabänder (T3 und T3 2 der Abb. 3) ein ${ }^{14} \mathrm{C}$-Alter zwischen $>8000$ und rund 12.000 a BP. Die Tephralagen T3, liegen im Hangenden des Tills der Moränengruppe M VI, während die Tephra- lagen $\mathrm{T} 3{ }_{2}$ im Liegenden des Tills M VI angetroffen werden. Die ${ }^{14} \mathrm{C}$-Alter für die Tephralagen T3 (Abb. 3) stammen aus Profilen im Bereich der Moränenwälle der Gruppe M V, wo infolge der Moränenwall-Topographie das Tephramaterial teilweise umgelagert wurde. Auf den Moränenwällen sind die Tephrahorizonte erodiert, während in den Senken zwischen den Moränenwällen das Tephramaterial in Wechsellagerung mit Mudden und Torfen abgelagert worden ist (Foto 3). Vermutlich verjüngten Umlagerungsprozesse und Durchwurzelung durch Riedgräser in den feuchten Senken zwischen den Moränenwällen die ${ }^{14} \mathrm{C}$ Alter. Bleiben die beiden kleinsten ${ }^{14} \mathrm{C}$-Daten unberücksichtigt (Kontamination infolge Durchwurzelung bei $\mathrm{Hv} 18070$ und 18071), ergeben sich Alter für die Tephralagen $\mathrm{T} 3$, die sich gut in die Chronostratigraphie einfügen (Abb. 3).

\subsection{Spätglaziale Gletschergeschichte ande- rer ecuadorianischer Vulkane (Pichincha, Chimborazo/Carihuairazo)}

Über die spätglazialen Gletscherbewegungen an den Vulkanen Pichincha und Chimborazo/Carihuairazo haben Heine \& HeIne (1996) und J.T. Heine $(1993,1995)$ berichtet. Die Ausführungen sollen hier nicht wiederholt werden.

Am Pichincha wird ein Gletschervorstoß belegt, der jünger als $10.600{ }^{14} \mathrm{C}$-Jahre $\mathrm{BP}$ und älter als $9000{ }^{14} \mathrm{C}$-Jahre BP ist (Abb. 4). Die Gletschergeschichte des Vulkans Guagua Pichincha und Rucu Pichincha wird von HeINE (1995a, 1995b) beschrieben. Kleine Kargletscher waren am Ende der YD und zu Beginn des Holozäns ausgebildet. Die Datierung ergibt sich aus tephrostratigraphischen Korrelierungen in Verbindung mit radiometrischen Altersbestimmungen von Tephrahorizonten und organischem Material in Mudden, Mooren und fossilen $\mathrm{A}_{\mathrm{h}}$-Horizonten der Andosols.

Der von Clapperton \& McEwan (1985) für das Chimborazo/Carihuairazo-Gebiet (Río MochaTal) postulierte Vorstoß eines YD-zeitlichen Gletschers wurde von J.T. HeINE $(1993,1995)$ kritisch beurteilt; J.T. HeINE (1993, 1995) belegt durch eine Neubearbeitung des Río Mocha-Profils und eine sorgfältige Deutung der Sedimente und glazialen Formen, daß zu keiner Zeit ein YD-zeitlicher Gletschervorstoß existierte. Dieser Interpretation schließen sich auch CLAPPERTON et al. (1997) zumindest indirekt - an, indem sie bei der Diskussion des YD-zeitlichen Gletschervorstoßes am 


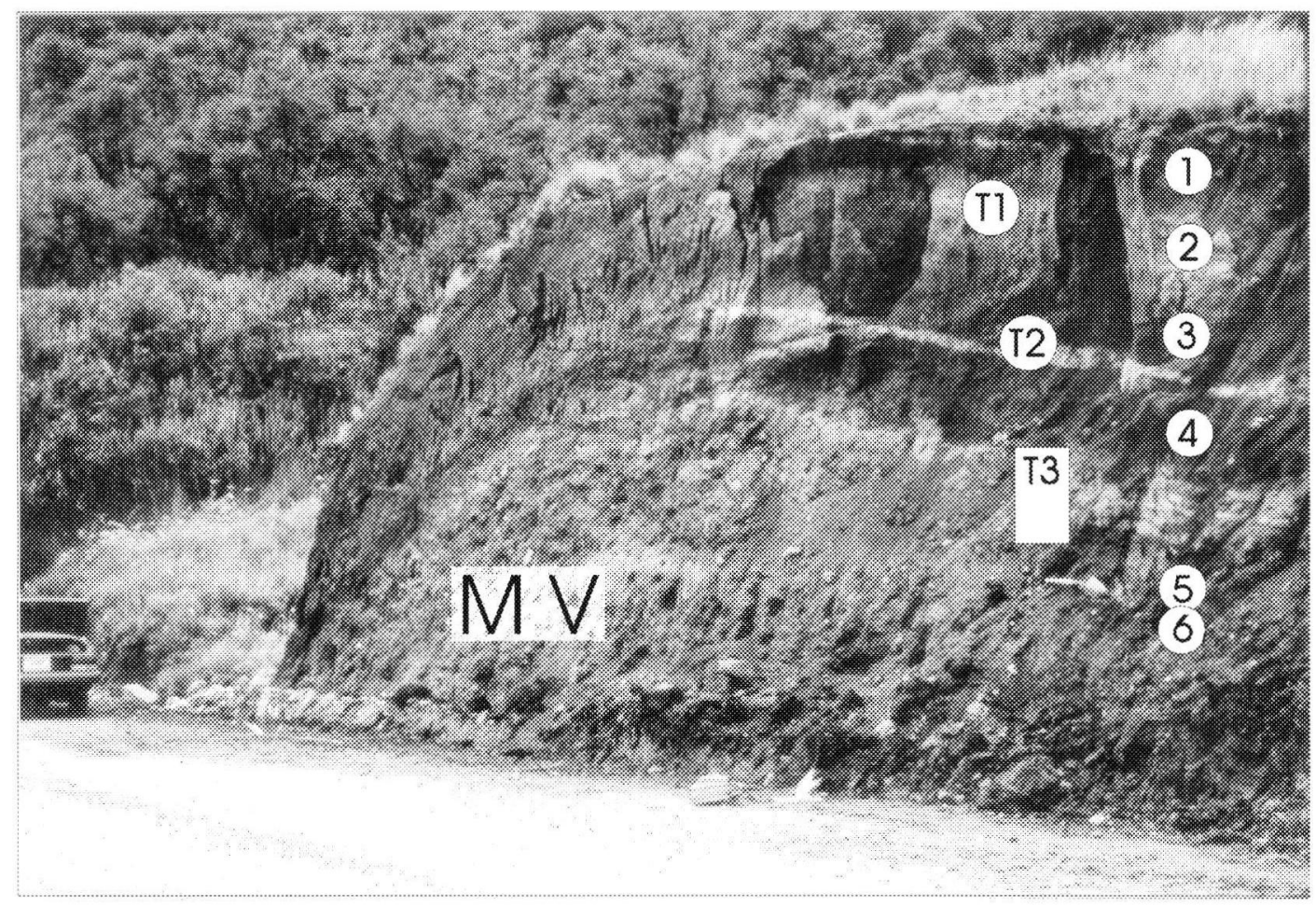

Foto 3: Papallacta-Gebiet. Moränenwall der Gruppe M V mit Deckschichten in $3870 \mathrm{~m}$ NN. Die Tephrahorizonte T1, T2 und T3 sind gekennzeichnet. Die Stellen der ${ }^{14} \mathrm{C}$-datierten Proben sind ebenfalls markiert: (1) ECU 117 (1070 \pm 50 -

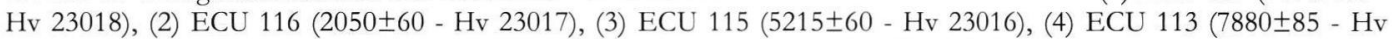

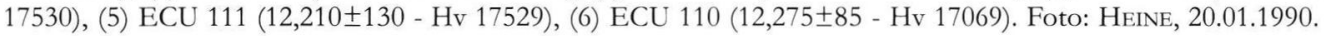

Foto 3: Papallacta area. Moraine wall of the group M V with tephra, peat and soil layers on top in $3870 \mathrm{~m}$ a.s.l. The tephra layers T1, T2 and T3 are marked. The location of the ${ }^{14} \mathrm{C}$ samples is also shown. (1) ECU 117 (1070 $\pm 50-\mathrm{Hv}$

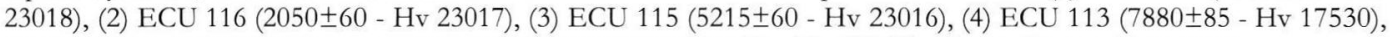

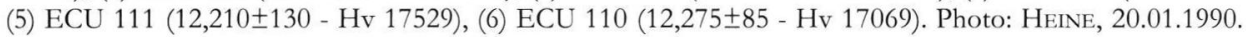

Papallacta-Paß nicht auf die Río Mocha-Situation eingehen, wo die Autoren doch zuvor immer diese Lokalität als Beleg für einen YD-zeitlichen Gletschervorstoß angeführt haben (z.B. ClaPPERTON 1993: 439-443).

\section{Interpretation und Diskussion}

Im Folgenden wird versucht, die Befunde hinsichtlich einer möglichst genauen Altersansprache zu diskutieren. Dabei sollen morphologische, paläopedologische, sedimentologische und tephrostratigraphische Analysen sowie ${ }^{14} \mathrm{C}$-Altersbestimmungen angewandt werden. Die Bezeichnungen der Moränengruppen, die älter als die YD sind, ist z.T. abweichend von früheren Angaben (z.B. HEINE 1995a, 1995b; HeInE \& HeINE 1996), da neue Forschungsergebnisse berücksichtigt werden.
Das Maximalalter des Gletschervorstoßes der Gruppe M VI ergibt sich aus dem Minimalalter der liegenden Schichten unter dem M VI-Till. Aus der Darstellung in Abbildung 3 wird ersichtlich, $\mathrm{da} ß$ die Sedimentation der Schichten unter dem M VI-Till ca. 1500 bis $2000{ }^{14} \mathrm{C}$-Jahre benötigte. Die Profile A und B (Abb. 3) lassen erkennen, daß wiederholt Abspülung und damit Umlagerung von Tephramaterial erfolgte. In Mulden (Profil A) wurde das abgespülte Material sedimentiert, am Hang (Profil B) konnte sich ein schwacher Boden entwickeln. Vor über rund $12.200{ }^{14} \mathrm{C}$-Jahren $\mathrm{BP}$ gab es keine Bodenbildung und auch keine Vegetationsentwicklung. Zwischen $12.140 \pm 80 \mathrm{a}$ BP und 10.265 165 a BP erfolgte die Ablagerung der Sandschichten und die Muddebildung unter dem M VI-Till. Die Alter von $10.555 \pm 140{ }^{14} \mathrm{C}$ Jahren BP und $10.265 \pm 165{ }^{14} \mathrm{C}$-Jahren BP stam- 


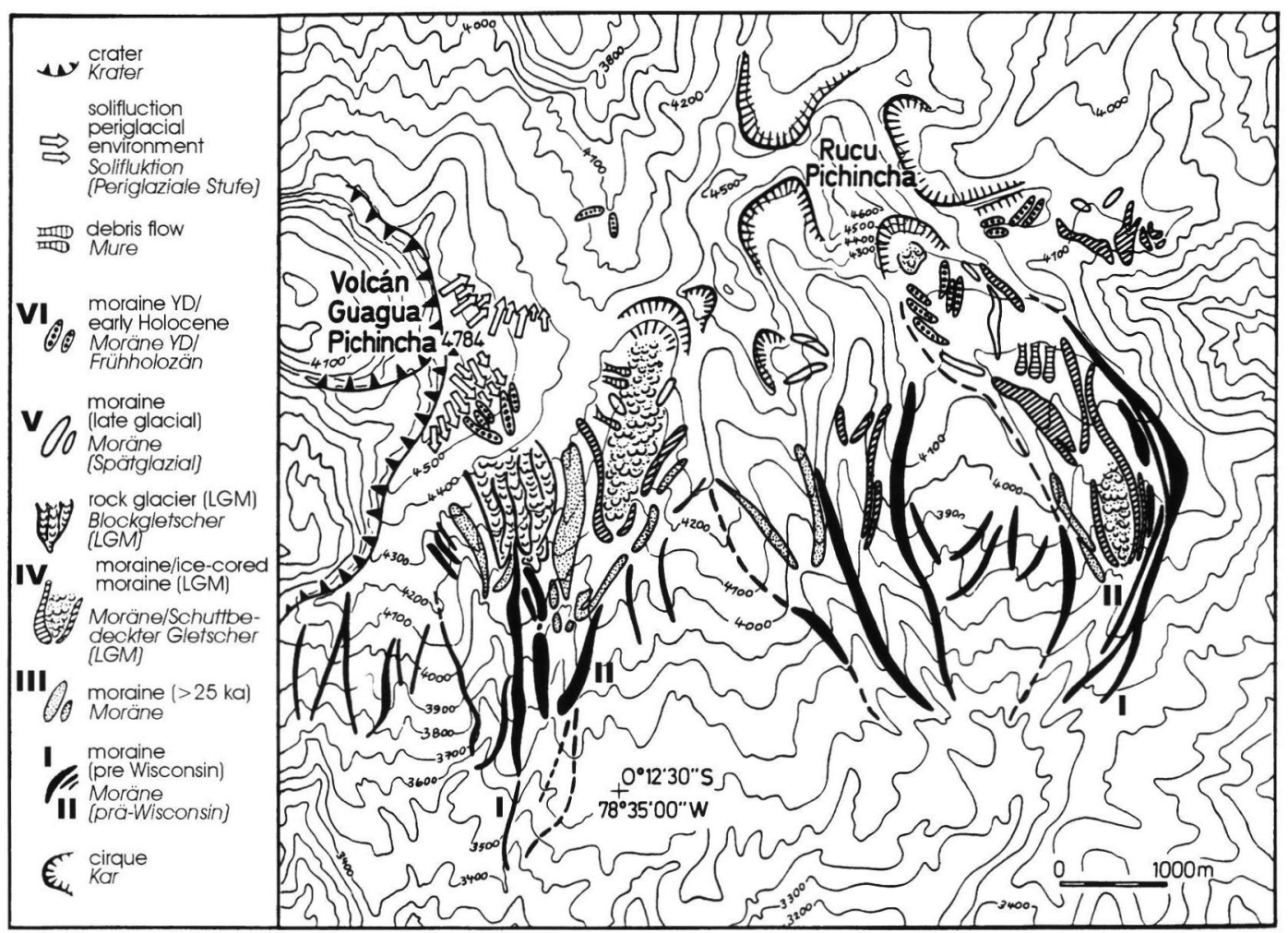

Abb. 4: Glazialmorphologische Skizze des Pichincha-Vulkangebirges (vgl. HeINE 1995a).

Fig. 4: Glacial sequence of Pichincha volcano (see HeIne 1995a).

men von Material, daß unmittelbar im Grenzbereich zwischen dem M VI-Till und den liegenden Sedimenten entnommen wurde. Die Alter dokumentieren somit das Maximalalter des Gletschervorstoßes, ebenso das von Clapperton et al. (1997) genannte Alter von $10.650 \pm 60{ }^{14} \mathrm{C}$-Jahren BP, das von einem Torfrest stammt, der im basalen Till eingeschlossen war.

Die ${ }^{14} \mathrm{C}$-Alter der Profile A und B sind chronologisch vernünftig. Es besteht kein Grund anzunehmen, daß sie nicht den Zeitabschnitt zwischen rund 12.200 und $10.300{ }^{14} \mathrm{C}$-Jahren BP repräsentieren. Da das Probenmaterial aus verschiedenen Horizonten entnommen wurde (mit Ausnahme der doppelten Datierung der Probe ECU 121), verbietet sich eine statistische Auswertung der Daten bezüglich einer Mittelwert-Berechnung des Alters (vgl. GEYH 2000). Wird allein die ${ }^{14} \mathrm{C}$-Zeitskala benutzt, dann würde das Maximalalter des Gletschervorstoßes M VI in die zweite Hälfte der YD fallen. Unter Berücksichtigung der kalibrierten ${ }^{14} \mathrm{C}$-Alter (Tab. 1) kann der Beginn des Vorstoßes M VI bereits zwischen $12.690-12.350 \mathrm{cal}$
BP (ECU 121) und 12.400-11.690 cal BP (ECU 186) liegen und damit sowohl in der ersten als auch in der zweiten Hälfte der YD.

Auch die ${ }^{14}$ C-Alter, die Clapperton et al. (1997) publizieren, können die Probleme der Datierung nicht lösen. Die Daten (Abb. 5) stimmen sehr gut mit den oben genannten ${ }^{14} \mathrm{C}$-Altern überein. Sie belegen ein Maximalalter des Gletschervorstoßes $\mathrm{M}$ VI von rund $10.700{ }^{14} \mathrm{C}$-Jahren $\mathrm{BP}$, was einem Alter von rund $12.600-12.700 \mathrm{cal}$ BP entspricht.

Das Minimalalter der Moränengruppe M VI konnte anhand der tephrostratigraphischen Arbeiten bisher nicht genau im Papallacta-Gebiet bestimmt werden. Aus den Profilen läßt sich ableiten, daß vor rund $8000{ }^{14} \mathrm{C}$-Jahren BP bereits eine intensive Bodenbildung auf den Deckschichten erfolgte. Welches Alter die unmittelbar über dem Till M VI liegenden Tephrahorizonte haben, konnte nicht ermittelt werden, da einerseits die ${ }^{14} \mathrm{C}$-Alter der entsprechenden Horizonte (Abb. 3) entweder infolge Kontamination (Wurzeln) nicht aussagekräftig und da andererseits einige Horizonte 
Tab. 1: ${ }^{14} \mathrm{C}$-Alter der Proben des Papallacta-Gebiets in Ecuador.

Tab. $1:{ }^{14} \mathrm{C}$ ages of samples from the Papallacta area in Ecuador.

\begin{tabular}{|c|c|c|c|c|c|}
\hline $\begin{array}{c}{ }^{14} \mathrm{C} \text { age } \\
\text { (a BP) } \\
{ }^{14} \mathrm{C}-\mathrm{Jahre} \text { BP }\end{array}$ & $\begin{array}{c}\text { Lab. No. } \\
\text { Hv }\end{array}$ & $\begin{array}{c}\text { Sample No. } \\
\text { Proben-Nr. } \\
\text { ECU }\end{array}$ & Material & $\begin{array}{l}\delta^{13} \mathrm{C} \\
(\% \circ)\end{array}$ & $\begin{array}{c}\text { cal age } \\
\text { cal. Alter } \\
\text { cal.... }\end{array}$ \\
\hline $255 \pm 60$ & 23025 & 130 & $\mathrm{P}$ & -25.1 & $\mathrm{AD} 1635-1954$ \\
\hline $555 \pm 60$ & 23026 & 131 & $\mathrm{P}$ & -25.8 & AD $1320-1430$ \\
\hline $680 \pm 90$ & 17066 & 132 & $\mathrm{P}$ & -27.4 & \\
\hline $920 \pm 70$ & 23027 & 133 & $P$ & -25.9 & $\mathrm{AD} 1025-1220$ \\
\hline $1070 \pm 50$ & 23018 & 117 & fAh & -24.9 & $\mathrm{AD} 900-1015$ \\
\hline $1405 \pm 70$ & 23028 & 134 & $\mathrm{P}$ & -26.0 & AD 605-675 \\
\hline $2050 \pm 60$ & 23017 & 116 & fAh & -25.1 & $\mathrm{BC} 115-\mathrm{AD} 20$ \\
\hline $4405 \pm 70$ & 17067 & 135 & fAh & -25.1 & \\
\hline $4680 \pm 110$ & 18070 & 173 & $\mathrm{P}, \mathrm{c}$ & -27.6 & \\
\hline $5215 \pm 60$ & 23016 & 115 & fAh & -25.4 & BC 4210-3965 \\
\hline $6795 \pm 65$ & 17064 & 100 & fAh & -26.3 & \\
\hline $7675 \pm 85$ & 17068 & 136 & fAh & -25.3 & \\
\hline $7880 \pm 85$ & 17530 & 113 & $\mathrm{G}$ & -26.7 & \\
\hline $9490 \pm 260$ & 18071 & 175 & $\mathrm{tR}$ & -29.6 & \\
\hline $10,265 \pm 165$ & 23040 & 186 & SOM & -27.4 & BC $10,400-9690$ \\
\hline $10,505 \pm 75$ & 17063 & 121 & $\mathrm{P}$ & -26.9 & \\
\hline $10,555 \pm 140$ & 23020 & 121 & $\mathrm{G}$ & -26.2 & BC $10,690-10,350$ \\
\hline $10,850 \pm 100$ & 18068 & 187 & $\mathrm{Pl}$ & -24.2 & \\
\hline $10,945 \pm 65$ & 24008 & 165 & $\mathrm{PH}$ & -25.8 & BC $10,990-10,835$ \\
\hline $11,150 \pm 160$ & 18780 & 164 & $\mathrm{PH}$ & -28.5 & \\
\hline $11,370 \pm 80$ & 23041 & 188 & fAh & -25.1 & BC $11,440-11,230$ \\
\hline $11,535 \pm 85$ & 23019 & 120 & G & -26.1 & BC 11,635-11,390 \\
\hline $11,765 \pm 155$ & 23042 & 190 & $\mathrm{sOM}$ & -26.4 & $\mathrm{BC} 11,975-11,570$ \\
\hline $11,770 \pm 90$ & 18072 & 176 & $\mathrm{P}$ & -28.6 & \\
\hline $12,140 \pm 80$ & 17062 & 118 & $\mathrm{P}$ & -27.4 & \\
\hline $12,210 \pm 130$ & 17529 & 111 & $\mathrm{G}$ & -28.0 & \\
\hline $12,250 \pm 130$ & 18069 & 170 & $\mathrm{P}$ & -26.9 & \\
\hline $12,275 \pm 85$ & 17069 & 110 & $\mathrm{P}$ & -26.9 & \\
\hline $12,330 \pm 95$ & 24009 & 167 & $\mathrm{HA}$ & -26.9 & BC $12,660-12,280$ \\
\hline
\end{tabular}

c = contaminated/kontaminiert

$\mathrm{fAh}=$ fossil Andosol, A horizon/fossiler Andosol, A-Horizont

$\mathrm{G}=$ gyttja/Gyttja

PH = plant material + humid acids/pflanzliches Material + Huminsäuren

$\mathrm{HA}=$ humid acid/Huminsäuren

$\mathrm{P} \quad=$ peat $/$ Torf

$\mathrm{Pl}=$ plants $/$ Pflanzen

$\mathrm{sOM}=$ sandy organic-rich material/Sand, reich an organischem Material

$\mathrm{tR} \quad=$ thin rootlets/feine $\mathrm{Wurzeln}$ 
Tab. 2: ${ }^{14} \mathrm{C}$-Alter theor. und Cal-Alter theor. für die Profile A und B der Fig. 3, berechnet aus den Sedimentationsraten. Tab. 2: ${ }^{14} \mathrm{C}$ ages theor. and cal ages theor. of the sections $\mathrm{A}$ and $\mathrm{B}$ of Figure 3 , calculated from sedimentation rates.

\begin{tabular}{|c|c|c|c|c|c|}
\hline $\begin{array}{l}\text { Depth beneath } \\
\text { till M VI } \\
\text { Tiefe unter } \\
\text { Till M VI } \\
\mathrm{cm} \\
\end{array}$ & $\begin{array}{l}\text { Sample No. } \\
\text { Proben-Nr. } \\
\text { ECU }\end{array}$ & $\begin{array}{l}{ }^{14} \mathrm{C} \text { age } \\
{ }^{14} \mathrm{C}-\text { Alter } \\
\text { a BP }\end{array}$ & $\begin{array}{l}\text { Cal. age } \\
\text { Kal. Alter } \\
\text { a cal BC }\end{array}$ & $\begin{array}{l}{ }^{14} \mathrm{C} \text { age theor. } \\
{ }^{14} \mathrm{C} \text {-Alter theor. } \\
\text { a BP }\end{array}$ & $\begin{array}{l}\text { Cal. age theor. } \\
\text { Kal. Alter theor. } \\
\text { a cal BC }\end{array}$ \\
\hline \multicolumn{6}{|l|}{ Profil A } \\
\hline 0 & 121 & $\begin{array}{l}10505 \pm 75 \\
10555 \pm 140\end{array}$ & $\begin{array}{l}10580-10355 \\
10690-10350\end{array}$ & $10560 \pm 60$ & $10595-10395$ \\
\hline 30 & 120 & $11535 \pm 85$ & $11640-11390$ & $11385 \pm 45$ & $11480-11325$ \\
\hline 40 & 119 & & & $11660 \pm 50$ & $11795-11615$ \\
\hline 60 & 118 & $12140 \pm 80$ & $12380-12060$ & $12205 \pm 75$ & $12445-12170$ \\
\hline \multicolumn{6}{|l|}{ Profil B } \\
\hline 0 & 186 & $10265 \pm 165$ & $10400-9690$ & $10290 \pm 131$ & $10435-10030$ \\
\hline 10 & 187 & $10850 \pm 100$ & $10935-10715$ & $10830 \pm 70$ & $10895-10695$ \\
\hline 20 & 188 & $11370 \pm 80$ & $11440-11230$ & $11375 \pm 75$ & $11455-11235$ \\
\hline 40 & 189 & & & $12465 \pm 225$ & $12810-12100$ \\
\hline
\end{tabular}

bisher nicht datiert worden sind. Allerdings nennen Clapperton et al. (1997) fünf ${ }^{14} \mathrm{C}$-Alter von Torf und Pflanzen-Makroresten aus den Deckschichten unmittelbar über dem Till M VI (Abb. 5). Die ${ }^{14} \mathrm{C}$-Alter deuten auf ein Minimumalter des Tills M VI von rund $10.000{ }^{14} \mathrm{C}$-Jahren BP bzw. von rund 11.000-11.600 cal BP. Damit würde die Zeit des Abschmelzens des Gletschers in das frühe Holozän fallen. Allerdings ist nicht ganz auszuschließen, daß das datierte Material hangabwärts verlagert wurde, so daß auch Bestandteile von älteren Humus- und Muddehorizonten in den datierten Proben enthalten sind.

Werden die Befunde anhand der konventionellen ${ }^{14} \mathrm{C}$-Alter diskutiert, so wird ein Gletschervorstoß belegt, der frühestens in der zweiten Hälfte der YD einsetzt, oder aber gegen Ende der YD, und der bis ins frühe Holozän existiert (vgl. Heine \& Heine1996). Auf gleiche Verhältnisse deuten auch die Ergebnisse vom Pichincha-Vulkangebirge hin (Abb. 4). Erst durch die Möglichkeit, die konventionellen ${ }^{14} \mathrm{C}$-Alter in kalibrierte ${ }^{14} \mathrm{C}$-Alter, die der Kalenderrechnung entsprechen, zu überführen, wirft erneut Fragen der Altersstellung der YDzeitlichen Gletscherbewegungen auf. Wenn die Datierung (ECU 186: 12.400-11.690 cal BP) aus den jüngsten Schichten unter dem Till M VI für die Ermittlung des Maximalalters des Gletschervorstoßes herangezogen wird, kann das Gletscher- vorrücken in die letzte Hälfte der YD gestellt werden.

Darauf deuten auch die Berechnungen der Tab. 2. Die ${ }^{14} \mathrm{C}$-Alter theor. und Cal-Alter theor. sind Rechenwerte, die sich aus den Sedimentationsraten der Profile A und B (siehe Abb. 3) ergeben. Die Sedimentationsraten betragen für das Profil A für die konventionellen ${ }^{14} \mathrm{C}$-Alter $0,036 \pm 0,002$ $\mathrm{cm} / \mathrm{a}$ und für die kalibrierten $0,033 \pm 0,003 \mathrm{~cm} / \mathrm{a}$. Der Unterschied erklärt sich aus der Verzerrung der ${ }^{14} \mathrm{C}$-Zeitskala. Mit diesen Sedimentationsraten wurden die konventionellen und kalibrierten Alter theor. für jede Schicht berechnet. Im Profil B betragen die Sedimentationsraten 0,018 $\pm 0,003$ $\mathrm{cm} / \mathrm{a}$ bzw. 0,018 $\pm 0,004 \mathrm{~cm} / \mathrm{a}$. Die berechneten Alter der Tabelle 2 deuten auf eine Bildung der humosen Schichten im Liegenden des Tills M VI bis in die zweite Hälfte der YD und damit auf einen entsprechenden spät-YD-zeitlichen Gletschervorstoß.

In Verbindung mit den Daten aus den hangenden Schichten (CLAPPERTON et al. 1997) (Abb. 5) kommt damit ein äußerst kurzer Zeitabschnitt in der zweiten Hälfte der YD und zu Beginn des Holozäns für den Gletschervorstoß in Frage. Die Größe des M VI-Gletschers im Papallacta-Gebiet widerspricht dieser Deutung nicht. 


\section{Papallacta Pass}

CLAPPERTON et al. (1997)

${ }^{14} \mathrm{C}$ a BP

\section{This study}

${ }^{14} \mathrm{C}$ a BP $\quad \mathrm{HV}$

$255 \pm 60 \quad 23025$

$555 \pm 60 \quad 23026$

$680 \pm 90 \quad 17066$

$920 \pm 70 \quad 23027$

$1070 \pm 50 \quad 23018$

$10,075 \pm 50$

$10,080 \pm 95$

$10,170 \pm 45$

$9770 \pm 60$

$9995 \pm 125$

$10,650 \pm 60$

$10,780 \pm 70$

$10,900 \pm 50$

$10,900 \pm 110$

$10,950 \pm 45$

$11,050 \pm 60$

$11,265 \pm 100$

$10,560 \pm 90$

$10,730 \pm 60$

$10,785 \pm 45$

$11,250 \pm 200$

$11,605 \pm 105$

$11,750 \pm 55$

$11,850 \pm 70$

$11,710 \pm 100$

$11,515 \pm 155$

$11,550 \pm 110$

$13,070 \pm 120-$

$13,010 \pm 50$

$12,940 \pm 60$

$13,095 \pm 50$

$13,160 \pm 115$

$13,220 \pm 90$

$13,190 \pm 70$

|||||||||| Andosol

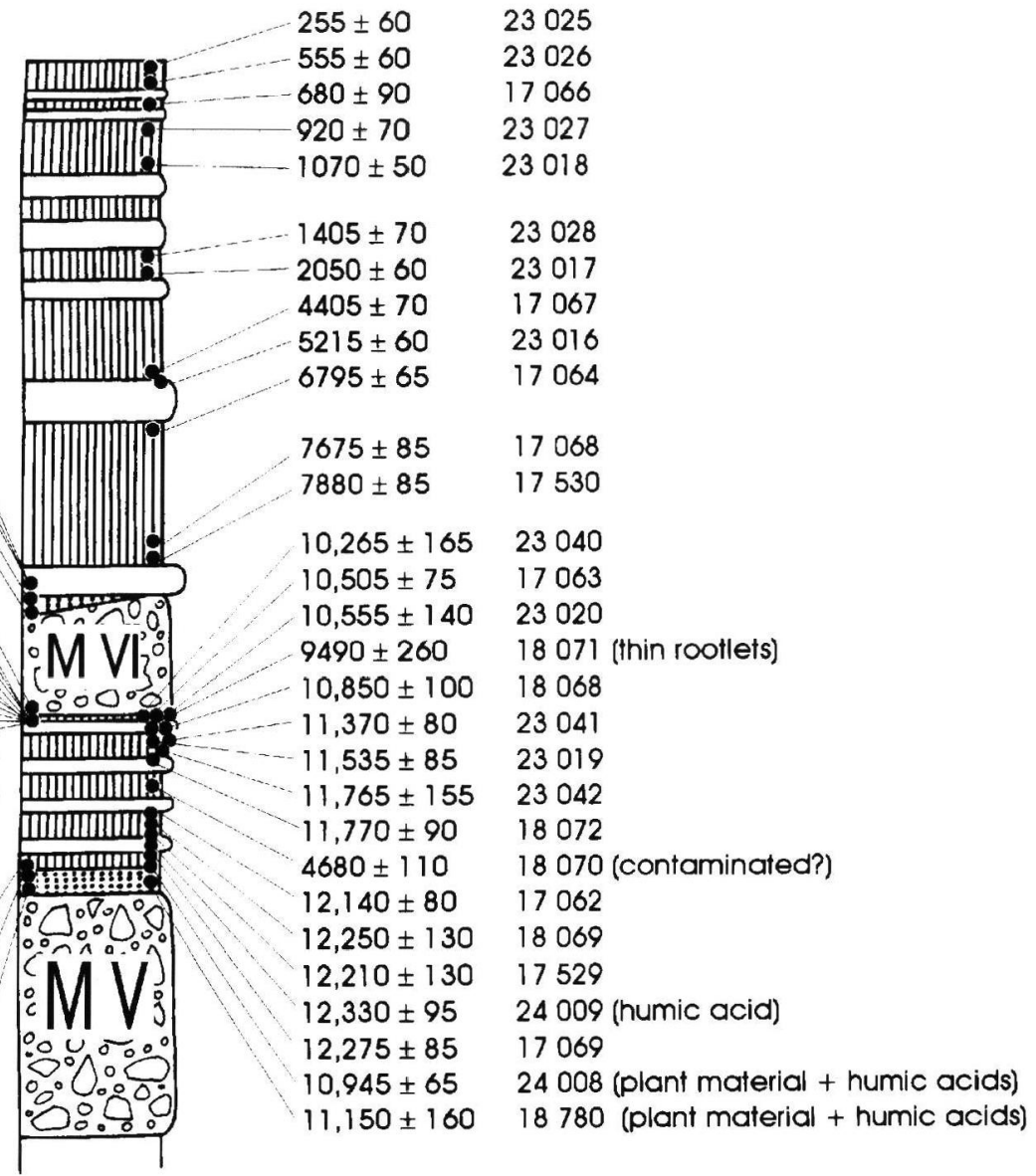

Tephra

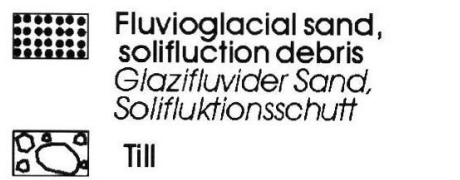

Abb 5: ${ }^{14} \mathrm{C}$-Alter der Papallacta-Pass-Profile nach Clapperton et al. (1997) und der vorliegenden Studie.

Fig. 5: ${ }^{14} \mathrm{C}$ ages of the Papallacta Pass sections published by CLAPPERTON et al. (1997) and in this study. 


\section{Korrelation in Südamerika}

Bereits vor Jahren publizierte J.T. HEINE (1992, 1993) eine kritische Würdigung der Belege für YD-zeitliche Gletscher- und Klimaschwankungen. Es stellt sich heraus, daß YD-zeitliche Gletschervorstöße in den südamerikanischen Anden nicht belegt werden können. Die Annahme der kalten und feuchten El Abra-Schwankung (VAN DER HAMMEN \& HOOGHIEMSTRA 1995; KuHRY et al. 1993; HoOghiemstra \& VAN Der HaMmen 1993) mit entsprechendem Gletschervorstoß während der YD muß bezweifelt werden, da die Datierungen unzureichend sind. Zweifellos gab es im Spätglazial Südamerikas Klimaschwankungen, die auch zu den Gletschervorstößen der Moränengruppen M V und M VI in Ecuador führten, jedoch gibt es bisher keinen zweifelsfreien Beleg für einen Gletschervorstoß im tropischen Südamerika, der genau mit der Klimaschwankung der YD zusammenfällt. In einer Zusammenstellung über glazialmorphologische Belege für YD-zeitliche Gletschervorstöße in den Kordilleren von Nord- und Südamerika können OsBorN et al. (1995) für Südamerika nur auf den Vorstoß eines ChimborazoGletschers im Río Mocha-Tal (Clapperton \& McEwAN 1985) verweisen, dessen Datierung jedoch von J.T. Heine $(1992,1993)$ in das Spätglazial vor $>13.000{ }^{14} \mathrm{C}$-Jahre $\mathrm{BP}$ vorgenommen wurde. RodBell \& SELTZER (2000) datieren ein schnelles Gletscherabschmelzen in der Cordillera Blanca (Peru) zu Beginn der YD als Folge der YDzeitlichen Niederschlagsabnahme. Für die YD können sie keinen Gletschervorstoß belegen (vgl. auch Seltzer 2001).

Die sorgfältige Aus- und Bewertung der Belege aus den tropischen Anden für eine YD-zeitliche Klimaschwankung zeigt, daß zwei Phasen mit Gletschervorstößen im Spätglazial/Frühholozän auftreten: $>13.000 / 12.000{ }^{14} \mathrm{C}$-Jahre BP und 10.000-9000 ${ }^{14}$ C-Jahre BP. Die mitgeteilten Befunde aus Ecuador dokumentieren sowohl einen Vorstoß, der älter als $12.500{ }^{14} \mathrm{C}$-Jahre BP ist (M V), als auch einen Vorstoß, der jünger als 10.500 ${ }^{14} \mathrm{C}$-Jahre BP ist. Allein die kritische Auswertung der Paläoklima-Archive (Moränensequenzen, Pollenprofile etc.) mit möglichst genauen Datierungen kann die eingangs aufgeworfenen Fragen nach dem Synchronismus oder dem Asynchronismus, dem regionalen und/oder globalen Auftreten, dem abrupten oder dem langsamen Wandel der YD beantworten helfen. Die Paläoklimatologie leidet unter dem Nachteil, daß diejenigen, die die Palä-
oklima-Archive beurteilen können (Palynologen, Sedimentologen etc.), normalerweise die Methoden der physikalischen und chemischen Altersbestimmungen zu wenig kennen, um zuverlässige Folgerungen ziehen zu können, und daß diejenigen, die die Methoden der Datierungen (Physiker, Chemiker etc.) und die Behandlung ihrer Ergebnisse beherrschen (Geochronologen, Physiker, Chemiker, Klimatologen etc.), die Geoarchive nicht zu deuten und zu bewerten vermögen.

Das Dilemma von Nachweisen für YD-zeitliche Gletscher und durch Pollenprofile belegte Klimaschwankungen in Südamerika ist zumindest in vielen Fällen in unzureichenden Datierungen der interpretierten Sedimente und Formen begründet. So lange beispielsweise Pollenprofile nur mit wenigen konventionellen ${ }^{14} \mathrm{C}$-Altern belegt sind (vgl. Hooghiemstra \& van der Hammen 1993: Abb. 4 bis 7; VAN 'T VEER et al. 2000) und diese zudem noch ohne Standardabweichungen für konkrete Altersansprachen angegeben werden, lassen sich die Probleme des Nachweises der YD nicht befriedigend lösen (vgl. dazu GEYH 2000). Bis auf den heutigen Tag werden in Lateinamerika Gletscher- und Klimaschwankungen ohne (oder aus Mangel an) hinreichende(n) Daten stillschweigend zeitlich korreliert, ohne daß für die vorgenommenen Korrelationen gesicherte wissenschaftliche Belege vorliegen (zuletzt BAKER et al. 2001:699 für die YD). Daraus können absonderliche wissenschaftliche Folgerungen resultieren (GEYH 2000), wie z.B. eine scheinbare Korrelation verschiedener spätglazialer Klimaschwankungen mit der YD im europäischen Sinne.

Eine kritische Durchsicht der Literatur hinsichtlich paläoklimatisch verwertbarer Belege für YDzeitliche Umweltveränderungen in Südamerika zeigt einige bemerkenswerte Aspekte. Abgesehen von den Vorstellungen von Colinvaux und Mitarbeitern (Colinvaux \& De Oliveira 2000; ColINVAUX et al. 2000), daß das tropische Südamerika während des LGM kühl-feuchte Verhältnisse aufwies, gehen heute fast alle Bearbeiter/innen von kühleren $\left(\right.$ ca. $5-7^{\circ} \mathrm{C}$ ) und trockeneren Umweltbedingungen für das tropische Südamerika aus (HEINe 2000; Thompson et al. 2000; Behling et al. 2000; Mayle 2000).

Heine \& Heine (1996) und Heine (2000) weisen darauf hin, daß die YD in den tropischen Anden im Vergleich zur Zeit davor und danach kühl und trocken war. Die geringeren Niederschläge wer- 


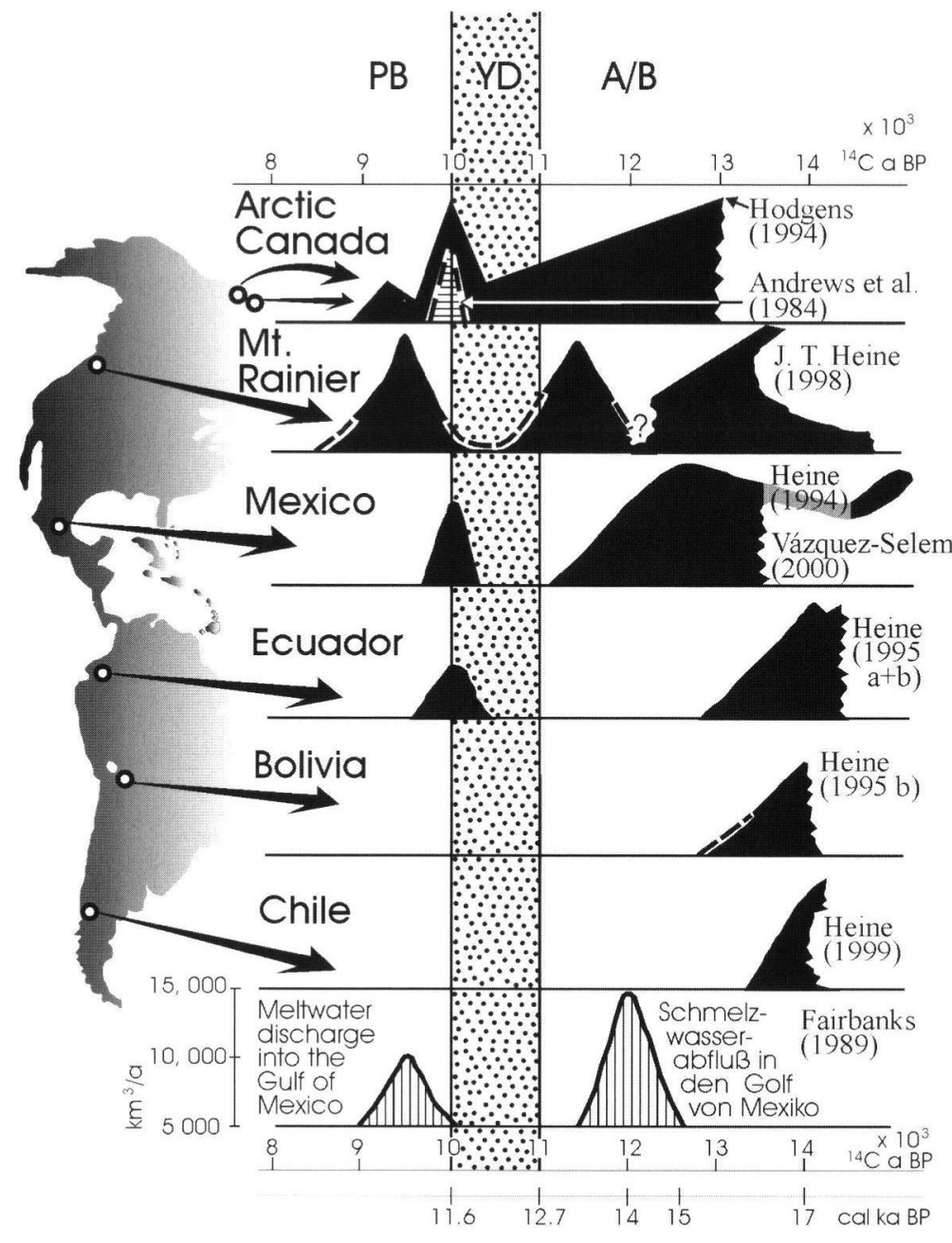

PB - Preboreal YD - Younger Dryas

A/B - Allerød/Bølling

Abb. 6: Spätglaziale Gletscherfluktuationen (ausgewählte Beispiele entlang der nord- und südamerikanischen (Kordilleren) und Schmelzwassererabfluß in den Golf von Mexiko.

Fig. 6: Late-glacial glacier fluctuations (selected examples of the North and South American Cordilleras) and meltwater discharge into the Gulf of Mexico.

den auch für das Ausbleiben der Gletschervorstöße verantwortlich gemacht. Ebenfalls geringere Niederschläge während der YD im Einzugsgebiet des Amazonas belegen die Ergebnisse vom Amazonas-Schwemmfächer (ODP site 942). MAsLIN et al. (2000) präsentieren eine detaillierte, gut datierte Sequenz mariner Sedimente, die belegt, daß während der YD die Abflußmenge des Amazonas um $40 \%$ zurückging als Folge geringerer Niederschläge in Amazonien. Um 10.200 ${ }^{14}$ C-Jah- re $\mathrm{BP}$ (ca. 11.400 cal BP), d.h. während Termination IB, tritt ein gewaltiger, jedoch kurzlebiger Gipfel in der AmazonasAbflußkurve auf, der in allen planktonischen und lithologisch/biogenetischen Archiven der Bohrkern-Sedimente deutlich hervortritt. Da mit diesem Ereignis auch ein vermehrter Eintrag von andinem Material über das AmazonasFlußsystem in den Atlantik verbunden ist, wird der $\mathrm{Ab}$ flußpeak einerseits dem Abschmelzen der Gletscher, andererseits höheren Niederschlägen zugeschrieben (MASLIN et al. 2000; vgl. auch SHOWERS \& BEVIS 1988). Hier findet sich der erste Anhaltspunkt dafür, daß die YD relativ trocken war und daß am Ende der YD abrupt eine starke Niederschlagszunahme in Amazonien und den tropischen Anden festzustellen ist. Dieses über die Meeressedimente sehr gut datierte Ereignis fällt mit dem plötzlichen Vorstoß der Gletscher der Gruppe M VI in Ecuador zusammen. Beide Ereignisse treten zur gleichen Zeit auf, sind von kurzer Dauer und dokumentieren hohe Niederschlagstätigkeit am Ende der YD. Das führt sowohl zum Gletschervorstoß als auch zum Abflußgipfel des Amazonas.

\section{Korrelation in Mittel- und Nordamerika sowie Europa}

Die Ausführungen zeigen, daß eine weltweite Korrelation der Paläoklima-Ereignisse nur dann vorgenommen werden darf, wenn die Datierung dieser Ereignisse hinreichend genau vorgenommen worden ist (Abb. 6).

Die Glazialgeschichte mexikanischer Vulkane im Spätglazial und Frühholozän beschreibt HeINE (1994). Die YD ist nicht durch ein Vorrücken der 
Gletscher belegt, wohl aber die Zeit unmittelbar danach. VÁZQUEZ SELEM (2000; vgl. auchVÁZQuEZ Selem \& Heine, im Druck) datiert in Mexiko am Nordhang des Iztaccíhuatl-Vulkanmassivs einen Gletschervorstoß auf $12.000-11.800 \mathrm{BP}\left({ }^{36} \mathrm{Cl}\right.$ exposure age), d.h. in die zweite Hälfte der YD. Damit wird deutlich, daß die YD wegen der gröBeren Aridität auch in Mexiko zu einem Gletscherverhalten führt, das zu Beginn der YD mit den aus Ecuador beschriebenen Verhältnissen vergleichbar ist. Ebenfalls größere Aridität wird aus anderen Gebieten Mittelamerikas während der YD berichtet, so für die Cordillera de Talamanca/ Costa Rica (Islebe et al. 1995).

Eine detaillierte Glazial-Chronostratigraphie vom Mount Rainier in Washington/USA J.T. HEINE 1998) belegt Gletschervorstöße vor $>11.300{ }^{14} \mathrm{C}$ Jahre BP (13.200 cal BP) und zwischen 9800 und $8950{ }^{14} \mathrm{C}$-Jahren BP (10.900 und $9950 \mathrm{cal} \mathrm{BP}$ ). Während der YD, zwischen 11.000 und 10.000 ${ }^{14} \mathrm{C}$-Jahren BP (12.900 und $11.600 \mathrm{cal} \mathrm{BP}$ ) schmolzen die Gletscher am Mount Rainier ab, vermutlich wegen Mangel an Feuchtigkeit bei ansonsten kalten Klimabedingungen. Auch hier zeigt sich, daß die Klimaschwankung der YD nachzuweisen ist, daß sie sich aber nicht durch ein Gletschervorrücken ausdrückt. Gletscherschwankungen wenn unzureichend genau datiert - würden im Falle der Prämisse, daß die YD zu Gletschervorstößen führte, am Mount Rainier zu einer falschen Alterseinschätzung führen.

In Skandinavien beispielsweise wird der YD-zeitliche Vorstoß der Gletscher auf der Basis von zahlreichen Chronostratigraphien aus verschiedenen Gebieten Norwegens auf 11.300 (Vorrücken) ${ }^{14} \mathrm{C}$-Jahre BP bis 10.300 (Abschmelzen) ${ }^{14} \mathrm{C}$-Jahre BP datiert (ANDERSEN et al. 1995), wobei die Möglichkeit nicht ausgeschlossen wird, daß es in Norwegen einen Vorstoß zu Beginn und einen zweiten am Ende der YD gegeben hat. In Finnland haben die Moränen des Salpausselkä I ein Alter von rund $11.000{ }^{14} \mathrm{C}$-Jahre BP, die von Salpausselkä II ein Alter von ca. $10.500{ }^{14} \mathrm{C}$-Jahre BP und die Moränen des CFIMF (= Central Finland IceMarginal Formation) von rund $10.000{ }^{14} \mathrm{C}$-Jahre BP (RAINIO et al. 1995). In Karelien ist die Situation noch unklar (Rainio et al. 1995). In Schweden wird versucht, die alten Warven-Chronologien in 'adjusted varve yr BP' zu überführen; die genaue Datierung verschiedener Moränenwälle in Mittelschweden und deren Korrelierung mit den norwegischen und finnischen YD-Moränen bereitet noch Schwierigkeiten (LUNDQUIST \& WOHLFARTH 2001).

Abschließend sei darauf hingewiesen, daß die YD auch als warme Episode in Erscheinung treten kann. Im subtropischen Florida belegen Pollenarchive, daß für mehrere gut datierte Ereignisse (z.B. H2 und H3) eine Erwärmung Floridas dem Eintrag von Eis-transportiertem Material in den Nord-Atlantik vorausging und daß die YD in Florida warm und feucht war (JACOBSON \& ALMQUISTJaCOBSON 2000). Diese Befunde werden durch die Ergebnisse von RÜHLEMANN et al. (1999) aus dem tropischen Atlantik ergänzt, die sowohl für Heinrich-Event $\mathrm{H} 1$ als auch für die YD (12.900-11.600 cal BP) dort eine signifikante Erwärmung belegen. Dies zeigt, daß Temperaturänderungen während der YD im tropischen Atlantik verglichen mit dem Nord-Atlantik nicht synchron (out of phase) verlaufen können. Als Erklärung dafür werden plötzliche Änderungen der thermohalinen Zirkulation vermutet.

Die wenigen Beispiele zum Gletscherverhalten während der YD geben Hinweise darauf, daß in den Gebieten bei Abkühlung und Niederschlagsabnahme (YD) kein Gletscherwachstum auftritt, in denen infolge relativ warmer und extrem feuchter Klimabedingungen (z.B. Ecuador, Mexiko, Mount Rainier/Washington) günstige Voraussetzungen für die Bildung von Gletschern herrschen. Die Gletscher rücken vor, wenn - oft bei relativ warmen Temperaturen am Übergang YD/Präboreal - eine starke Niederschlagszunahme zu verzeichnen ist (Abb. 6). Damit eignen sich kleine Gletscher nicht zu chronostratigraphischen Korrelierungen über weite Entfernungen (vgl. HeINE 1983).

\section{Zusammenfassung und Würdigung}

Am Papallacta-Paß in der Ostkordillere von Ecuador haben Moränen ein Alter, das einen Gletschervorstoß am Ende der YD und zu Beginn des Holozäns belegt. Während der ersten Hälfte der YD waren in Ecuador - wie auch in anderen Gebieten des tropischen Südamerika - die Niederschläge zu gering, um markante Gletschervorstöße auszulösen. Erst über eine möglichst exakte Datierung der glazialen Sedimente kann die YD sowie die Zeit davor und danach hinreichend genau über glazialmorphologische Paläoklima-Archive zeitlich eingestuft (korreliert) werden. Un- 
ter Hinzuziehung ausschließlich der PaläoklimaBelege, die exakt datiert sind, läßt sich die YD im tropischen Südamerika grob paläoklimatisch rekonstruieren: Die YD war kühler und zugleich trockener als die Abschnitte davor und danach. Gegen Ende der YD oder am Übergang von der YD zum Holozän zeichnet sich eine sprunghafte (abrupte) Niederschlagszunahme ab, deren Höhepunkt von kurzer Dauer ist (vermutlich nur wenige Jahrhunderte oder gar Jahrzehnte).

Da viele Paläoklima-Archive paläoklimatisch differenziert interpretiert und hypothetisch bestimmten Zeitabschnitten des Spätpleistozäns und Holozäns zugeordnet werden, ohne daß hierfür hinreichende Voraussetzungen durch genaue Datierungen vorliegen, resultieren daraus oft absonderliche wissenschaftliche Folgerungen (GEYH 2000). Fortschritte auf dem Gebiet der Paläo-Umweltforschung einschließlich der Rekonstruktion der spätglazialen abrupten Klimaoszillationen sind nur möglich, wenn verstärkt den Datierungsfragen Aufmerksamkeit geschenkt wird (vgl. ANDrES \& LITT 2000) und weniger versucht wird, immer mehr 'neue' - schlecht datierte - Befunde dem bereits bestehenden - oft revisionsbedürftigen Paläoklima-Weltbild hinzuzugesellen. Die Erkenntnisse werden dadurch nicht besser, im Gegenteil, sie werden nur scheinbar bestätigt (z.B. OsBorn et al. 1995).

\section{Danksagung}

Die Arbeiten wurden von der Deutschen Forschungsgemeinschaft, der VW-Stiftung und der Universität Regensburg finanziell gefördert. Dafür danken wir. Dank gebührt auch vielen Kolleginnen und Kollegen für Anregungen und Diskussionen sowie zahlreichen Mitarbeitern für Beistand im Gelände und im Labor. Stellvertretend für alle möchten wir nennen: J.T. HEINE (Seattle) für hilfreiche Anmerkungen im Gelände und bei Auswertungen, A. HEINE für Unterstützung aller logistischen Arbeiten.

\section{Schriftenverzeichnis}

Andersen, B.G., Mangerud, J., Sörensen, R., Reite, A., Sveian, H., Thoresen, M. \& Bergström, B. (1995): Younger Dryas ice-marginal deposits in Norway. - Quaternary International, 28: 147-169; Oxford.

Anderson, D.E. (1997): Younger Dryas research and its implications for understanding abrupt climatic change. - Progress Physical Geography, 21: 230249; London.
Andres, W. \& LitT, T. (2000): Termination I in Central Europe. - Quaternary International, 61: 1-4; Oxford.

Andrews, J.T., Miller, G.H., Vincent, J-S. \& Shilts, W.W. (1984): Quaternary correlations in Arctic Canada. - In: R.J. Fulton [Ed.], Quaternary Stratigraphy of Canada - A Canadian Contribution to IGCP Project 24. Geol. Survey of Canada Paper, 84-10: 127-134; Ottawa.

Baker, P.A., Rigsby, C.A., Seltzer, G.O., Fritz, S.C., Lowenstein, T.K., Bacher, N.P. \& Veliz, C. (2001): Tropical climate changes at millennial and orbital timescales on the Bolivian Altiplano. - Nature, 409: 698-701; London.

Behling, H., Arz, H.W., Pätzold, J. \& Wefer, G. (2000): Late Quaternary vegetational and climate dynamics in northeastern Brazil, inferences from marine core GeoB 3104-1. - Quaternary Science Reviews, 19: 981-994; Oxford.

Clapperton, C.M. (1985): Significance of a Late-Glacial readvance in the Ecuadorian Andes. - Quaternary of South America and Antarctic Peninsula, 3: 149-158; Rotterdam (Balkema).

Clapperton, C.M. (1987a): Glacial geomorphology, Quaternary glacial sequence and palaeoclimatic inferences in the Ecuadorian Andes. - In: V. GARDINER [Ed.]: International Geomorphology 1986 Part II: 843-870; London (Wiley).

Clapperton, C.M. (1987b): Maximal extent of late Wisconsin glaciation in the Ecuadorian Andes. - Quaternary of South America and Antarctic Peninsula, 5: 165-179; Rotterdam (Balkema).

Clapperton, C.M. (1990): Glacial and volcanic geomorphology of the Chimborazo-Carihuairazo Massif, Ecuadorian Andes. - Transactions Royal Society Edingburgh: Earth Sciences, 1: 91-116; Edingburgh.

Clapperton, C.M. (1993): Quaternary Geology and Geomorphology of South America. - 779 S.; Amsterdam (Elsevier).

Clapperton, C.M., Hall, M., Mothes, P., Hole, M.J., Still, J.W., Helmens, K.F., Kuhry, P. \& A.M.D. Gemmell (1997): A Younger Dryas Icecap in the Equatorial Andes. - Quaternary Research, 47: 1328; San Diego.

Clapperton, C.M. \& McEwan, C. (1985): Late Quaternary moraines in the Chimborazo area, Ecuador. - Arctic and Alpine Research, 17: 135-142; Boulder.

Clark, P.U., Clague, J.J., Curry, B.B., Dreimanis, A., Hicock, S.R., Miller, G.H., Berger, G.W., Eyles, N., Lamothe, M., Miler, B.B., Mott, R.J., OldaLE, R.N., Stea, R.R., SZabo, J.P., Thorleifson, L.H. \& VINCENT, J.-S. (1993): Initiation and development of the Laurentide and Cordilleran ice sheets following the last Interglaciation. - Quaternary Science Reviews, 12: 79-114; Oxford.

Colinvaux, P. \& De Oliveira, P.E. (2000): Palaeoecology and climate of the Amazon basin during the last glacial cycle. - Journal of Quaternary Science, 15: 347-356; Chichester. 
Colinvaux, P., De Oliveira, P.E. \& Bush, M.B. (2000): Amazonian and neotropical plant communities on glacial time-scales: The failure of the aridity and refuge hypotheses. - Quaternary Science Reviews, 19: 141-169; Oxford.

Colman, S.M., Keigwin, L.D. \& Forester, R.M. (1994): Two episodes of meltwater influx from glacial Lake Agassiz into the Lake Michigan basin and their climatic contrasts. - Geology, 22: 547-550; Boulder.

Denton, G.H. (2000): Inter-Hemispheric linkage of palaeoclimate during the last termination. - Quaternary International, 63/64: 48-49 (INQUA Conf. Abstr.); Oxford.

Denton, G.H. \& Hendy, C.H. (1994): Younger Dryas Age Advance of Franz Josef Glacier in the Southern Alps of New Zealand. - Science, 264: 1434-1437; Washington.

Denton, G,H., Heusser, C.J., Lowell, T.V., Moreno, P.I., Andersen, B.G., Heusser, L.E., Schlóchter, C. \& Marchant, D.R. (1999): Interhemispheric linkage of paleoclimate during the last glaciation. Geografisca Annaler, 81 A: 107-153; Oxford.

de Vernal, A., Hillaire-Marcel, C. \& Bllodeau, G. (1996): Reduced meltwater outflow from the Laurentide ice margin during the Younger Dryas. Nature, 381: 774-777; London.

FAIRBANKS, R.G. (1989): A 17,000-year glacio-eustatic sea level record: influence of glacial melting rates on the Younger Dryas event and deep-ocean circulation. - Nature, 342: 637-642; London.

GEYH, M.A. (2000): Zum Umgang mit numerischen Altersangaben. - In: VÖLKEL, J. \& BARTH, H.-J. [Eds.], Beiträge zur Quartärforschung, Regensburger geogr. Schriften, 33: 1-17; Regensburg.

Gillespie, A. \& Molnar, P. (1995): Asynchronous maximum advances of mountain and continental glaciers. - Reviews of Geophysics, 33: 311-364.

Goslar, T., Arnold, M., Tisnerat-Laborde, N., CzerNIK, J. \& WIECKOWSKI, K. (2000): Variations of Younger Dryas atmospheric radiocarbon explicable without ocean circulation changes. - Nature, 403: 877-880; London.

Hastenrath, S. (1981): The Glaciation of the Ecuadorian Andes. - 159 S.; Rotterdam (Balkema).

HeINE, J.T. (1992): Younger Dryas - Did it happen in South America? - Unveröff. Dipl-Arbeit, Geogr. Inst. Universität Bonn: 107 S.; Bonn.

HeInE, J.T. (1993): A reevaluation of the evidence for a Younger Dryas climatic reversal in the tropical Andes. - Quaternary Science Reviews, 12: 769-779; Oxford.

Heine, J.T. (1995): Comments on C. M. Clapperton's „Glacier readvances in the Andes at 12 500-10000 yr BP: implications for mechanism of Late-glacial climatic change (JQS 8, 197-215)“. - Journal of Quaternary Science, 10: 87-88; Chichester.

Heine, J.T. (1998): Extent, timing, and climatic implications of glacier advances, Mount Rainier, Washington, U.S.A., at Pleistocene/Holocene transition. - Quaternary Science Reviews, 17: 1139-1148.

HEINE, K. (1983): Führt die Quartärforschung zu nichtaktualistischen Modellvorstellungen in der Geo- morphologie? - Colloquium Geographicum, 16: 93 121; Bonn (Dümmler).

Heine, K. (1994): The late-glacial moraine sequences in Mexico: is there evidence for the Younger Dryas event? - Palaeogeography, Palaeoclimatology, Palaeoecology, 112: 113-123; Amsterdam.

Heine, K. (1995a): Late Quaternary glacier advances in the Ecuadorian Andes: A preliminary report. Quaternary of South America and Antarctic Peninsula, 9: 1-22; Rotterdam (Balkema).

Heine, K. (1995b): Bedded Slope Deposits with Respect to the Late Quaternary Glacial Sequence in the High Andes of Ecuador and Bolivia. - In: SLAYMAKER, O. [Ed.]: Steepland Geomorphology: $257-$ 278; Chichester (Wiley).

Heine, K. (1999): Der Kleine Süden Chiles - eine ,klassische" Glaziallandschaft. Neue Feldforschungen und Ergebnisse zum Problem der interhemisphärischen Korrelation jungpleistozäner glazialer Ereignisse. - Bamberger geogr. Schr., 19: 77-105; Bamberg.

HeIne, K. (2000): Tropical South America during the Last Glacial Maximum: evidence from glacial, periglacial and fluvial records. - Quaternary International, 72: 7-21; Oxford.

Heine, K. \& Heine, J.T. (1996): Late Glacial Climatic Fluctuations in Ecuador: Glacier Retreat during Younger Dryas Time. - Arctic and Alpine Research, 28: 496-501; Boulder.

Hodgson, D.A. (1994): Episodic ice streams and ice shelves during retreat of the northwesternmost sector of the late Wisconsinan Laurentide Ice Sheet over the central Canadian Arctic Archipelago. Boreas, 23: 14-28; Oslo.

Hooghiemstra, H. \& van der Hammen, T. (1993): Late Quaternary vegetation history and paleoecology of Laguna Pedro Palo (subandean forest belt, Eastern Cordillera, Colombia). - Review Palaeobotany Palynology, 77: 235-262; Amsterdam.

Hostetler, S.W., Bartlein, P.J., Clark, P.U., Small, E.E. \& Solomon, A.M. (2000): Simulated influences of Lake Agassiz on the climate of central North America 11,000 years ago. - Nature, 405: 334-337; London.

Islebe, G.A., Hooghiemstra, H. \& van der Borg, K. (1995): A cooling event during the Younger Dryas Chron in Costa Rica. - Palaeogeography, Palaeoclimatology, Palaeoecology, 117: 73-80; Amsterdam. Jacobson, G.L. \& Almouist-Jacobson, H: (2000): A warm, moist Younger Dryas in subtropical Florida, USA. - Quaternary International, 63/64: 64 (INQUA Conf. Abstr.); Oxford.

Jouzel, J., Vaikmae, R., Petit, J.R., Martin, M., Duclos, Y., Stievenard, M., Lorius, C., Toots, M., Mélières, M.A., Burckle, L.H., Barkov, N.I. \& Kotlyakov, V.M. (1995): The two-step shape and timing of the last deglaciation in Antarctica. - Climate Dynamics, 11: 151-161; Berlin, Heidelberg.

Kaser, G., Hastenrath, S. \& Ames, A. (1996a): Mass balance profiles on tropical glaciers. - Zeitschrift Gletscherkunde u. Glazialgeologie, 32: 75-81; Innsbruck. 
Kaser, G., Georges, Ch., Ames, A. (1996b): Modern glacier fluctuations in the Huascarán-Chopicalqui massif of the Cordillera Blanca, Perú. - Zeitschrift Gletscherkunde u. Glazialgeologie, 32: 91-99; Innsbruck.

Kuhry, P., Hooghiemstra, H., van Gel, B. \& van der Hammen, T. (1993): The El Abra Stadial in the Eastern Cordillera of Colombia (South America). - Quaternary Science Reviews, 12: 333-343; Oxford.

Lauer, W. \& D. RAFiqPoor (1986): Geoökologische Studien in Ecuador. - Erdkunde, 40: 68-72; Bonn.

Lundquist, J. \& Wohlfarth, B. (2001): Timing and east-west correlation of south Swedish ice marginal lines during the Late Weichselian. - Quaternary Science Reviews, 20: 1127-1148; Amsterdam.

Maslin, M.A., Durham, E., Burns, S.J., Platzman, E., Grootes, P., Greig, S.E.J., Nadeau, M-J., SchleIcher, M., Pflaumann, U., Lomax, B. \& RimingTON, N. (2000): Palaeoreconstruction of the Amazon River freshwater and sediment discharge using sediments recovered at Site 942 on the Amazon Fan. - Journal of Quaternary Science, 15: 419-434; Chichester.

MAyle, F.E. (2000): Late Quaternary history of the Amazon forest/savanna boundary, NE Bolivia. Quaternary International, 63/64: 93 (INQUA Conf. Abstr.); Oxford.

Osborn, G., Clapperton, C.M., Thompson Davis, P., Reasoner, M., Rodbell, D.T., Seltzer, G.O. \& ZielinsKI, G. (1995): Potential glacial evidence for the Younger Dryas event in the Cordillera of North and South America. - Quaternary Science Reviews, 14: 823-832; Oxford.

Rainio, H., SAARnisto, M. \& EkMAN, I. (1995): Younger Dryas end moraines in Finland and NW Russia. - Quaternary International, 28: 179-192; Oxford.

Raynaud, D., Barnola, J.-M., Chappellaz, J., Blunier, T., Indermühle, A. \& Stauffer, B. (2000): The ice record of greenhouse gases: a view in the context of future changes. - Quaternary Science Reviews, 19: 9-17; Oxford.

Rodbell, D.T. \& SELTzer, G.O. (2000): Rapid Ice Margin Fluctuations during the Younger Dryas in the Tropical Andes. - Quaternary Research, 54: 328338; San Diego.

Rühlemann, C., Mulitza, S., Müller, P.J., Wefer, G. \& ZAHN, R. (1999): Warming of the tropical Atlantic Ocean and slowdown of thermohaline circulation during the last deglaciation. - Nature, 402: 511-514; London.

SAuER, W. (1971): Geologie von Ecuador. - 316 S.; Berlin, Stuttgart (Born-traeger).

Seltzer, G. O. (2001): Paleoclimates of the Central Andes. - PAGES News, 9(1): 16; Bern.

Showers, W.J. \& Bevis, M. (1988): Amazon cone isotopic stratigraphy: evidence for the source of the tropical fresh-water spike. - Palaeogeography, $\mathrm{Pa}$ laeoecology, Palaeoclimatology, 64: 189-199; Amsterdam.
Sikes, E.L., Samson, C.R., Guilderson, T.P. \& Howard, W.R. (2000): Old radiocarbon ages in the southwest Pacific Ocean during the last glacial period and deglaciation. - Nature, 405: 555-559; London.

Taylor, K.C., Lamorey, G.W., Doyle, G.A., Alley, R.B., Grootes, P.M., Mayewsini, P.A., White, J.W.C. \& BARLOW, L.K. (1993): The flickering switch of Late Pleistocene climate change. - Nature, 361: 432-436; London.

Thompson, L.G., Mosley-Thompson, E. Henderson, K.A. (2000): Ice-core palaeoclimate records in tropical South America since the Last Glacial Maximum. - Journal of Quaternary Science, 15: 377394; Chichester.

van der Hammen, T. \& Hooghiemstra, H. (1995): The El Abra Stadial, a Younger Dryas Equivalent in Colombia. - Quaternary Science Reviews, 14: 841 851; Oxford.

Van 'T Veer, R., Islebe, G.A. \& Hooghiemstra, H. (2000): Climatic change during the Younger Dryas chron in northern South America: a test of the evidence. - Quaternary Science Reviews, 19: 18211835; Amsterdam.

VÁZQUEZ SELEM, L. (2000): Glacial chronology of Iztaccíhuatl volcano, central Mexico. A record of environmental change on the border of the tropics. - Ph.D. Thesis, Arizona State University, 257pp.

VÁzquez Selem, L. \& Heine, K. (im Druck): Late Quaternary Glaciation of Mexico. - In: EHLERs, J. \& GibBard, P. I. [Hrsg.]: Extent and Chronology of Glaciations, vol. 3 (Asia, Africa, Latin America, Australia, Antarctica), Elsevier, Amsterdam.

Wolf, T. (1892): Geografía y Geología del Ecuador. 671 S.; Leipzig (Brockhaus). 